\title{
Geometry of Self-Similar Sets
}

\author{
Kristine A. Roinestad \\ Master's Thesis submitted to the Faculty of the \\ Virginia Polytechnic Institute and State University \\ in partial fulfillment of the requirements for the degree of
}

Master of Science

in

Mathematics

Dr. Peter Haskell, Chair

Dr. Martin Day

Dr. Jim Thomson

May 8, 2007

Blacksburg, Virginia

Keywords: Self-Similarity, Bilipschitz Equivalence, Cantor Sets

Copyright 2007, Kristine A. Roinestad 


\title{
Geometry of Self-Similar Sets
}

\author{
Kristine Roinestad
}

(ABSTRACT)

This paper examines self-similar sets and some of their properties, including the natural equivalence relation found in bilipschitz equivalence. Both dimension and preservation of paths are determined to be invariant under this equivalence. Also, sophisticated techniques, one involving the use of directed graphs, show the equivalence of two spaces. 


\section{Acknowledgments}

I am grateful for the support and encouragement of my thesis advisor, Dr. Peter Haskell, who patiently, but firmly, directed my progress, bolstered my confidence, and helped bring my research and study skills to a higher level. My sincere appreciation also extends to Dr. Martin Day and Dr. Jim Thomson, members of my Masters Thesis Committee, for their time and effort in reviewing this work. 


\section{Contents}

1 Introduction $\quad 1$

2 Hausdorff Dimension $\quad 4$

3 Properties of Bilipschitz Maps 11

4 Ultrametric Spaces $\quad 14$

$\begin{array}{lll}5 & \text { Self-Similar Sets } & 17\end{array}$

6 Bilipschitz Equivalence of Cantor Sets $\quad 32$

$\begin{array}{lll}7 & \text { A Surprising Equivalence } & 37\end{array}$

8 Bilipschitz Equivalence of Cantor Sets Continued 48

9 A Look Ahead $\quad 52$ 


\section{List of Figures}

5.1 First Stage in Sierpinski Carpet Formation . . . . . . . . . . . . . . . 21

7.1 Directed Graph for Cantor Set . . . . . . . . . . . . . . . . . . 39

7.2 Directed Graph $G^{*} \ldots \ldots \ldots \ldots \ldots$

7.3 Directed Graph $H^{*} \ldots \ldots \ldots \ldots \ldots$ 


\section{Chapter 1}

\section{Introduction}

For many students of mathematics, their introduction to the standard middle thirds Cantor set is in an undergraduate real analysis course. From the instructor's syllabus, they might realize the basics, that the set not only has standard Lesbesgue measure zero, but that it is also uncountable. They soon discover they are in for a wild and curious ride through the counter-intuitive and sometimes strange Cantor set and other self-similar sets.

In addition to Cantor sets, this paper delves into a more general class of sets, self-similar sets. A self-similar set is a metric space that is the union of scaled versions of itself, with scaling factor less than one. In looking at self-similarity, we will be focusing on a property of Euclidean spaces that is used frequently in analysis. When we blow up a neighborhood of a point to see how tangent lines approximate the graph of $y=f(x)$, we invert a distancedecreasing scaling map. The reason we can view a derivative as a linear map is because of 
the self-similarity of the vector space $\mathbb{R}^{n}$. Every open ball centered at a given point can be described in the same terms, for example, in terms of a fixed basis with a scaling of distance relating one ball to another.

We will be studying different self-similar sets and one important aspect of their geometry, bilipschitz equivalence. This is a natural notion of equivalence for self-similar sets. Sets are bilipschitz equivalent if the mapping from one set onto the other does not expand or contract distances by more than a constant factor. Since this equivalence gives positive bounds on distances, most estimates relevant for analysis, such as those appearing in definition of continuity, that hold on a space hold on any bilipschitz equivalent space. As with many equivalence relations, it may be easier to gather information on one particular representative of an equivalence class. Once we understand one representative of a class, we then understand all members of that class.

This paper considers Hausdorff measure, which leads to investigating the Hausdorff dimension, an invariant under bilipschitz equivalence. Equality of Hausdorff dimensions does not imply that two sets are bilipschitz equivalent, as examples will show. However, if two sets are equivalent they have the same dimension.

Also, we will show that the presence of paths is preserved under bilipschitz equivalence. The presence of paths is a very important property in proving two spaces are not bilipschitz equivalent. Using this tool, we can show that two sets, even with the same Hausdorff dimension, are not equivalent if one has at least one nonconstant path and another does not. 
There are also more sophisticated methods of showing equivalences, and in some cases surprising equivalences. One of these methods involves the use of directed graphs. For more restricted classes of spaces we are more likely to find invariants that guarantee two spaces are bilipschitz equivalent. This will be shown in a theorem that gives necessary and sufficient conditions for a self-similar set to be bilipschitz equivalent to a Cantor set.

This paper illustrates that although the complexities of self-similar sets, such as the standard Cantor middle third set, can be considered whimsical and unexpected, more importantly, they express the beauty of the language of mathematics and clearly demonstrate that sometimes the more you learn, the more you question. 


\section{Chapter 2}

\section{Hausdorff Dimension}

The discussion starts with the definition of Hausdorff dimension. First, however, a few definitions need to be stated.

Definition 1 If $(M, d)$ is a metric space and $E \subseteq M$ then the diameter of $E$, denoted $\operatorname{diam} E$ is given by $\operatorname{diam} E=\sup \{d(x, y) \mid x, y \in E\}$.

Definition 2 Let $(N, \rho)$ be a metric space and $A \subseteq N$. The d-dimensional Hausdorff measure of the set $A$ is defined as $H^{d}(A)=\lim _{\delta \rightarrow 0} H_{\delta}^{d}(A)$ where, for any $\delta>0$,

$$
H_{\delta}^{d}(A)=\inf \left\{\Sigma \operatorname{diam}\left(E_{j}\right)^{d} \mid E_{j} \subseteq A, A \subseteq \cup_{j=1}^{\infty} E_{j}, \operatorname{diam}\left(E_{j}\right)<\delta\right\}
$$

It is convenient to call a cover of $E$ by countably many sets, each of diameter less than $\delta$, a $\delta$ cover of $E$. 
A natural question, when first given the definition of Hausdorff measure, is: Does such an infimum exist? And, the answer is yes, the infimum does definitively exist. All expressions involved are nonnegative. Hence, we are looking at a non-empty subset of the extended reals that is bounded below. As $\delta$ decreases the number of possible covers of a set $A$ decreases. Thus we see that $H_{\delta}^{d}(A)$ grows monotonically as $\delta \rightarrow 0$. This shows that $H^{d}(A)$ must in fact exist, although it is possible that the limit is $\infty$, by an analogue of the monotone convergence theorem. Note that because the limit as $\delta \rightarrow 0$ is associated with a monotone function of $\delta$, to calculate our limit it suffices to consider any $\delta_{n} \rightarrow 0$. Also note that the map that takes $s$ to $H^{s}(A)$ is non-increasing.

It turns out that if $H^{s}(E)<\infty$ then for $t>s$ we have $H^{t}(E)=0$. Assume that $H^{s}(E)<\infty$ and $t>s$. Then, for $E_{j}$ with $\operatorname{diam}\left(E_{j}\right) \leq \delta$, we see $\Sigma\left(\operatorname{diam} E_{j}\right)^{t} \leq \delta^{t-s} \Sigma\left(\operatorname{diam} E_{j}\right)^{s}$. Thus, as $\delta \rightarrow 0$ we get that $H^{t}(E)=0$. Similarly if $t>s$ and $H^{t}(A)>0$ then $H^{s}(A)=\infty$. Thus, it follows that $\left\{s \mid H^{s}(E)=\infty\right\}$ and $\left\{s \mid H^{s}(E)=0\right\}$ are intervals that, when both nonempty, have a single point in the intersection of their closure. This observation permits two equivalent definitions of Hausdorff dimension.

\section{Definition 3 A set $E$ has Hausdorff dimension $d$ if}

$d=\inf \left\{s \mid H^{s}(E)=0\right\}=\sup \left\{s \mid H^{s}(E)=\infty\right\}$.

If $s<d$, then $H^{s}(E)=\infty$ and if $s>d$, then $H^{s}(E)=0$. It is usually easy to show that the dimension of a set $E$ is less than or equal to $d$. It suffices to find, for each $s>d$, some finite $k \in \mathbb{R}$ such that, for arbitrarily small $\delta$, there exists a $\delta$ cover of $E,\left\{E_{j}\right\}$, with 
$\Sigma\left(\operatorname{diam}\left(E_{j}\right)\right)^{s}<k$. For such $s$ and $k, H^{s}(E)=\lim _{\delta \rightarrow 0}\left\{\Sigma\left(\operatorname{diam}\left(E_{j}\right)\right)^{s} \mid \operatorname{diam}\left(E_{j}\right)<\delta\right\} \leq k$.

To show that the dimension of a set $E$ is greater than or equal to $d$, it suffices to show, for every $s<d$, that there is a $l>0$ such that for every cover $\left\{E_{j}\right\}, \Sigma_{j}\left(\operatorname{diam}\left(E_{j}\right)\right)^{s} \geq l$. For such $s$ and $l, H^{s}(E)=\lim _{\delta \rightarrow 0} \inf \Sigma_{j}\left(\operatorname{diam}\left(E_{j}\right)\right)^{s} \geq l$. In some cases one can prove the dimension $d$ of a set $E$, by reasoning as before, but for $s=d$ we need only show that $0<l \leq H^{d}(E) \leq k<\infty$. Finding the exact Hausdorff d-dimensional measure of a set of Hausdorff dimension $d$ requires a lot of work; however, it is Hausdorff dimension, not the Hausdorff measure that is crucial in this paper and so we will not calculate the latter. To see an example of a rigorous calculation of Hausdorff measure, refer to [4].

Example 1 The Hausdorff dimension of $[a, b] \subseteq \mathbb{R}$ is 1 .

\section{Proof:}

Suppose, for an arbitrary $\delta>0$, that $\left\{I_{j}\right\}$ is a countable collection of subsets of diameter less than $\delta$ such that $[a, b] \subseteq \cup_{j} I_{j}$. We may assume that each $I_{j}$ is a closed subinterval.

Choose $\epsilon>0$. For each $j$ extend $I_{j}$ on each side to make a new closed interval $I_{j}^{\prime}$ satisfying $l\left(I_{j}^{\prime}\right)-l\left(I_{j}\right) \leq \frac{\epsilon}{2^{j}}$ and $l\left(I_{j}^{\prime}\right)<\delta$ where $l(I)$ represents the length of the interval $I$. The interiors of the $I_{j}^{\prime}$ 's cover $[a, b]$ and by compactness we may choose a finite subcover $I_{j_{1}}^{\prime}, \ldots, I_{j_{n}}^{\prime}$. By a finite covering argument we get $l\left(I_{j_{1}}^{\prime}\right)+\ldots+l\left(I_{j_{n}}^{\prime}\right) \geq b-a$ and hence $\Sigma_{j} l\left(I_{j}^{\prime}\right) \geq b-a$. Thus we have $\Sigma_{j} l\left(I_{j}\right) \geq b-a-\epsilon$. Since $\epsilon>0$ was arbitrary we have $H^{1}([a, b]) \geq b-a>0$. 
Now, for our arbitrary $\delta>0$ choose $n \in \mathbb{N}$ so that $\frac{b-a}{n} \leq \frac{\delta}{2}$. Partition $[a, b]$ into $n$ subintervals of length $\frac{b-a}{n}$. Call these intervals $\left\{E_{j}\right\}$. Thus, $H_{\delta}^{1}([a, b])=\lim _{\delta \rightarrow 0} \Sigma_{j} \operatorname{diam}\left(E_{j}\right) \leq \Sigma_{1}^{n} \frac{b-a}{n}=$ $n \times \frac{b-a}{n}=b-a$. So, we have that $H^{1}([a, b]) \leq b-a<\infty$.

Thus we have shown that the Hausdorff dimension of $[a, b]$ is 1 and $H^{1}([a, b])=b-a$, the length of our interval.

Example 2 The Hausdorff dimension of $[a, b] \times[a, b] \subseteq \mathbb{R}^{2}$ is 2 .

\section{Proof:}

Take $\delta>0$. Cover $[a, b] \times[a, b]$ by arbitrary sets of diameter less than $\delta$. These sets might as well be closed balls, call them $\left\{B_{j}\right\}$. For each ball $B_{j}$ there exists $k_{j}$ so that $\frac{b-a}{2^{k_{j}+1}} \leq \operatorname{diam}\left(B_{j}\right)<\frac{b-a}{2^{k_{j}}}$

Choose $\epsilon>0$. Expand $B_{j}$ to a closed ball $B_{j}^{\prime}$ so that $\operatorname{diam}\left(B_{j}^{\prime}\right)$ satisfies $\frac{b-a}{2^{k_{j}+1}} \leq \operatorname{diam}\left(B_{j}^{\prime}\right)<$ $\frac{b-a}{2^{k_{j}}}$ and so that $\left(\operatorname{diam}\left(B_{j}^{\prime}\right)\right)^{2}-\left(\operatorname{diam}\left(B_{j}\right)\right)^{2} \leq \frac{\epsilon}{2^{j}}$. This is possible by the continuity of squaring.

The interiors of the $B_{j}^{\prime}$ 's form an open cover of $[a, b] \times[a, b]$ and thus by compactness we may choose a finite subcover and return to closed balls. Let $\left\{B_{j_{1}}^{\prime}, \ldots B_{j_{n}}^{\prime}\right\}$ denote the collection of closed balls.

Let $k_{0}=\max _{j_{1}, \ldots, j_{n}} k_{j}$ which were chosen above. Note that if $\frac{b-a}{2^{k+1}} \leq \operatorname{diam}\left(B_{j}^{\prime}\right)<\frac{b-a}{2^{k}}$ then $B_{j}^{\prime}$ can intersect a most four of the interiors of the squares in the standard decomposition into squares of side length $\frac{b-a}{2^{k_{j}}}$. Each $B_{j}^{\prime}$ can intersect at most $4^{k_{0}-k_{j}}=2^{2\left(k_{0}-k_{j}\right)}$ of the interiors 
of squares in the standard decomposition of our given square into squares of side length $\frac{b-a}{2^{k_{0}}}$. Note,

$$
2^{2\left(k_{0}-k_{j}\right)}=2^{2 k_{0}} 2^{-2 k_{j}} \leq 2^{2 k_{0}} \frac{2^{2}}{(b-a)^{2}}\left(\operatorname{diam}\left(B_{j}^{\prime}\right)\right)^{2} .
$$

Because our finite cover has to hit every one of the interiors of the $2^{2 k_{0}}$ squares in the $\frac{b-a}{k_{0}}$ decomposition,

$$
2^{2 k_{0}} \leq \Sigma_{j \in j_{1}, \ldots j_{n}} 2^{2 k_{0}} \frac{2^{2}}{(b-a)^{2}}\left(\operatorname{diam}\left(B_{j}^{\prime}\right)\right)^{2}
$$

So,

$$
\frac{(b-a)^{2}}{4} \leq \Sigma_{j}\left(\operatorname{diam}\left(B_{j}^{\prime}\right)\right)^{2} \leq \Sigma_{j}\left(\operatorname{diam}\left(B_{j}\right)\right)^{2}+\epsilon
$$

Thus, since $\epsilon$ was arbitrary, $H^{2}([a, b] \times[a, b]) \geq \frac{(b-a)^{2}}{4}>0$.

For our arbitrary $\delta>0$, choose $n \in \mathbb{N}$ such that $\frac{b-a}{n} \sqrt{2} \leq \frac{\delta}{2}$. Partition $[a, b]$ into $n$ subintervals of length $\frac{b-a}{n}$. Then we can form $n^{2}$ open squares of diameter $\frac{b-a}{n} \sqrt{2}$ to cover $[a, b] \times[a, b]$. $H_{\delta}^{2}([a, b] \times[a, b]) \leq \Sigma_{1}^{n^{2}}\left(\frac{b-a}{n}\right)^{2}=n^{2} \times\left(\frac{b-a}{n}\right)^{2}=(b-a)^{2}<\infty$.

Thus $0<H^{2}([a, b] \times[a, b])<\infty$ and we have that $[a, b] \times[a, b]$ has Hausdorff dimension 2 . 
Example 3 The Hausdorff dimension of a rectifiable simple non-closed path is 1.

\section{Proof:}

[4] Let $C$ be a simple non-closed path. Let $C_{x, y}$ denote the part of $C$ between $x$ and $y$. Now, orthogonal projection onto the line does not increase distances we see that $H^{1}\left(C_{x, y}\right) \geq$ $H^{1}([x, y])=|x-y|$, where $[x, y]$ is the straight line segment connecting $x$ and $y$.

Let $x_{1}, x_{2}, \ldots, x_{m}$ be a partition of $C$. If we can show that $H^{1}(C) \geq \Sigma_{1}^{m} C\left(x_{i}, x_{i+1}\right)$ then we would have $H^{1}(C) \geq \Sigma_{1}^{m}\left|x_{i+1}-x_{i}\right|>0$.

To show $H^{1}(C) \geq \sum_{1}^{m} H^{1}\left(C\left(x_{i}, x_{i+1}\right)\right)$ we can find open balls as small as necessary around the $x_{i}$ 's so that we have a disjoint union of finitely many compact sets $\left\{E_{j}\right\}$. For such disjoint unions we have $H^{1}\left(\cup_{j} E_{j}\right)=\Sigma_{j} H^{1}\left(E_{j}\right)$ since there is a finite distance between any two distinct $E_{j}$ and hence as $\delta \rightarrow 0$ the calculations separate. The $\epsilon$-errors introduced can have two possible effects, one is on $H^{1}(C)$ and the other on the images of the projection to segments $\Sigma_{1}^{m}\left|x_{i+1}-x_{i}\right|$. (Note, $2 \epsilon$ errors for arbitrarily small $\epsilon$ is fine).

Thus we have that $H^{1}(C) \geq \Sigma_{1}^{m}\left|x_{i+1}-x_{i}\right|>0$.

Now, let $l(C)$ denote the length of $C$. Let $f:[0, l(C)] \rightarrow C$ be the mapping that takes a point $t$ to a point on $C$ at distance $t$ from one of its distinct ends. Since $|f(t)-f(u)| \leq|t-u|$ we get from Lemma 1 given in the following chapter, that $H^{1}(C) \leq l(C)<\infty$.

Thus we have $0<H^{1}(C)<\infty$ and hence the Hausdorff dimension of $C$ is 1 . 
The next two examples follow from the definition of Hausdorff measure and the fact that the Hausdorff dimension of $[a, b]$ is 1 .

Example $4 H^{\frac{1}{2}}([a, b])=\infty$.

Example $5 H^{\frac{3}{2}}([a, b])=0$.

Now, we will move on to bilipschitz maps. 


\section{Chapter 3}

\section{Properties of Bilipschitz Maps}

Definition 4 Let $(M, d(x, y))$ and $(N, \rho(u, v))$ be metric spaces. A mapping $f: M \rightarrow N$ is said to be Lipschitz if there is a constant $C$ such that $\rho(f(x), f(y)) \leq C d(x, y)$ for all $x, y \in M$. We will say $f$ is $\mathbf{C}$-Lipschitz to make the constant explicit. We say $f$ is bilipschitz if there is a $C$ such that

$$
C^{-1} d(x, y) \leq \rho(f(x), f(y)) \leq C d(x, y)
$$

for all $x, y \in M$. We will say $f$ is $\mathbf{C}$-bilipschitz to be more explicit.

A Lipschitz map does not expand distances by more than a constant factor. Thus a Lipschitz map is a stronger form of uniform continuity. Now, a bilipschitz map does not expand or contract distances by more than a constant factor. Hence, bilipschitz maps are not only a stronger form of uniform continuity, but they also have uniformly continuous inverses. 
Definition 5 Two metric spaces $(M, d)$ and $(N, \rho)$ are bilipschitz equivalent if there exists a bilipschitz map $f$ of $(M, d)$ onto $(N, \rho)$.

Lemma 1 [1] If $f: M \rightarrow N$ is $K$ - Lipschitz then $H^{d}(f(A)) \leq K^{d} H^{d}(A)$.

\section{Proof:}

If $\left\{E_{j}\right\}$ is a $\delta$-cover for $A$, then $\Sigma \operatorname{diam} f\left(E_{j}\right)^{d} \leq \Sigma\left(K \operatorname{diam}\left(E_{j}\right)\right)^{d}=K^{d} \Sigma \operatorname{diam}\left(\left(E_{j}\right)\right)^{d}$ since $f$ is $K-$ Lipschitz. Let $\nu=K \delta$. Now $\left\{f\left(E_{j}\right)\right\}$ is a $\nu$ cover for $f(A)$. Thus, $H_{\nu}^{d}(f(A)) \leq$ $K^{d} H_{\delta}^{d}(A)$. Since $\nu \rightarrow 0$ as $\delta \rightarrow 0$, thus $H^{d}(f(A)) \leq K^{d} H^{d}(A)$. [4] $\square$

Corollary 1 [1] If $f: M \rightarrow N$ is $K$-bilipschitz then $K^{-d} H^{d}(A) \leq H^{d}(f(A)) \leq K^{d} H^{d}(A)$

\section{Proof:}

Apply Lemma 1 to $f$ and $f^{-1}$.

This shows that Hausdorff dimension is invariant under bilipschitz equivalence. Hausdorff dimension is not the only invariant under bilipschitz equivalence. It turns out that the presence of rectifiable paths is also an invariant of bilipschitz equivalent. 
Proposition 1 The presence of continuous paths is preserved under bilipschitz equivalence.

\section{Proof:}

Let $(M, d)$ and $(N, \rho)$ be bilipschitz equivalent metric spaces. Let $f: M \rightarrow N$ be a bilipschitz map of $M$ onto $N$. Let $\gamma$ be a continuous path in $M$. Then $f \circ \gamma$ is a continuous path in $N$ as desired.

Now we know that if two metric spaces have different Hausdorff dimension, they cannot be bilipschitz equivalent. Also, we know that if one metric space has a rectifiable path, where as another metric space does not, they as well cannot be bilipschitz equivalent. 


\section{Chapter 4}

\section{Ultrametric Spaces}

In many cases, one can show that a particular representative of an equivalence class has a property or characteristic that extends to the entire equivalence class. This can be an effective technique to prove characteristics of many self-similar sets. We will use this technique to show that Cantor sets, which are defined below, have no nonconstant paths. This is not clear for all such examples, so to start, we will note that many self-similar sets, such as Cantor sets, are bilipschitz equivalent to ultrametric spaces. We will then show that ultrametric spaces have no nonconstant paths, and thus Cantor sets have no nonconstant paths. Now, let us define an ultrametric space.

Definition 6 A metric space $(M, d)$ is an ultrametric space if for all $x, y, z \in M$ the metric satisfies $d(x, z) \leq \max \{d(x, y), d(y, z)\}$. 
Example 6 The middle thirds Cantor set is bilipschitz equivalent to an ultrametric space

This will be shown in chapter 5 .

Definition $7 A$ open ball centered at $x$ of radius $r$ in a metric space $(M, d)$ is $B(x, r)=$ $\{y \mid d(x, y)<r\}$

The next result says that in an ultrametric space if two open balls intersect, then one is contained inside the other.

Proposition 2 For any ultrametric space $(M, d)$ if $B(x, r) \cap B(y, t) \neq \emptyset$ and $r \leq t$ then $B(x, r) \subseteq B(y, t)$

\section{Proof:}

Let $(M, d)$ be an ultrametric space and let $z \in B(x, r) \cap B(y, t)$. Take $w \in B(x, r)$. Then $d(w, z) \leq \max \{d(w, x), d(z, x)\}<r$. Then we get $d(w, y) \leq \max \{d(w, z), d(z, y)\}<$ $\max \{r, t\}=t$. Thus, $w \in B(y, t)$ and $B(x, r) \subseteq B(y, t)$ as desired. $\square$

Note, that if $B(x, r) \cap B(y, r) \neq \emptyset$ then the two balls are equal. Thus we know that in any open ball $B$ in an ultrametric space all $x \in B$ can be considered the center of $B$.

There are many more properties of interest to us involving paths, one of which is discussed below. 
Proposition 3 In an ultrametric space there are no nonconstant paths.

\section{Proof:}

Suppose that there is a nonconstant path in the ultrametric space $(M, d)$. Let $f:[0,1] \rightarrow M$ be this nonconstant path. Let $f(0) \neq f(1)$. Choose $\epsilon$ such that $0<\epsilon<\frac{1}{2} d(f(0), f(1))$. By uniform continuity of $f$ choose $\delta$ such that $d(x, y)<\delta$ gives $d(f(x), f(y))<\epsilon$. Partition $[0,1]$ into closed subintervals of length less than $\delta$, so $[0,1]=\cup_{l=0}^{n-1}\left[x_{i}, x_{i+1}\right]$ where $x_{0}=0$ and $x_{n}=1$ and for all $i\left|x_{i}-x_{i+1}\right|<\delta$. By definition $f(0) \in B_{\epsilon}(f(0))$. Now, since $\left|x_{0}-x_{1}\right|<\delta$ we have that $d\left(f(0), f\left(x_{1}\right)\right)<\epsilon$. Thus we know $f\left(x_{1}\right) \in B_{\epsilon}(f(0))$.

By Proposition $3 B_{\epsilon}\left(f\left(x_{1}\right)\right) \subseteq B_{\epsilon}(f(0))$. Similarly one can show that $B_{\epsilon}\left(f\left(x_{i}\right)\right) \subseteq B_{\epsilon}\left(f\left(x_{i-1}\right)\right) \subseteq$ $\ldots \subseteq B_{\epsilon}(f(0))$. Thus for $i=n$ we have $B_{\epsilon}\left(f\left(x_{n}\right)\right) \subseteq B_{\epsilon}(f(0))$. Since $x_{n}=1$ we have $B_{\epsilon}(f(1)) \subseteq B_{\epsilon}(f(0))$. However, we chose $\epsilon$ so that $\epsilon<\frac{1}{2} d(f(0), f(1))$. So we have $\epsilon<\frac{1}{2} d(f(0), f(1))<\epsilon$, a contradiction. Thus there are no nonconstant paths in an ultrametric space. 


\section{Chapter 5}

\section{Self-Similar Sets}

We will now study self-similar sets. A self-similar set, which is defined explicitly below, is a metric space that is the union of scaled versions of itself, with scaling factor less than one (with scale factors arbitrarily small.) This is a great visualization technique. Moreover, in looking at self-similarity, we are focusing on an important property of Euclidean spaces that is used frequently in analysis. When we blow up a neighborhood of a point to see how tangent lines approximate the graph of $y=f(x)$, we are inverting a contractive similitude, defined below. The fact that we can view a derivative as a linear map is due to the self-similarity of the vector space $\mathbb{R}^{n}$. This is basically because we can get the entire local story by picking a basis and changing only the length.

Now, let us look at a self-similar set, and then we will give an explicit definition of selfsimilarity. 
Example 7 The standard middle thirds Cantor set is a self-similar set.

The way to create this set is to begin with the closed unit interval, $[0,1]$. Now, set $C_{1}=$ $[0,1]-\left(\frac{1}{3}, \frac{2}{3}\right)$, that is, remove the open middle third of $[0,1]$. Set $C_{2}=C_{1}-\left\{\left(\frac{1}{9}, \frac{2}{9}\right) \cup\left(\frac{7}{9}, \frac{8}{9}\right)\right\}$. So to form $C_{2}$ we remove an open middle third from each subinterval in $C_{1}$. Continue this process by induction, so that $C_{n}$ is $C_{n-1}-I_{n}$ where $I_{n}$ is the collection of open middle thirds of subintervals in $C_{n-1}$. Now the standard middle third Cantor set, $C$, is the intersection of all $C_{n}$. Thus $C=\cap_{n=1}^{\infty} C_{n}$. Now, look at the two functions $f_{1}(x)=\frac{1}{3} x$ and $f_{2}(x)=\frac{1}{3} x+\frac{2}{3}$. Note that $C=\cup_{1}^{2} f_{i}(C)$. This will prove that $C$ is a self-similar set in the sense of the following definitions.

Definition 8 A mapping $f: \mathbb{R}^{n} \rightarrow \mathbb{R}^{n}$ is a contractive similitude or a contraction if $r d(x, y)=d(f(x), f(y))$, where $d$ is the Euclidean metric, and $r<1$ is the contraction ratio or similitude ratio.

Definition 9 [2]Let $\left\{f_{k}\right\}_{k=1}^{N}$, mapping $\mathbb{R}^{n}$ into itself, be a finite family of contractive similitudes. A compact, nonempty set $E$ satisfying $E=\cup_{k=1}^{n} f_{k}(E)$ is called the invariant set or the self-similar set of $\left\{f_{k}\right\}_{k=1}^{n}$.

We will show in Proposition 4 that such invariant sets exist and are unique.

Definition 10 [3] A set of contractions $\left\{\phi_{i}\right\}_{i=1}^{m}$ is called an iterated function scheme. 


\section{Example 7 Continued}

Since $C=\cup_{i=1}^{2} f_{i}(C)$ and $f_{i}$ is clearly a contractive similitude with contraction ratio $\frac{1}{3}$ we have that the standard middle thirds Cantor set is the invariant set for the iterated function scheme $\left\{f_{1}, f_{2}\right\}$.

Many people do not think of a self-similar set in terms of unions, but instead in terms of intersections, such as in the formation of the Cantor set given above. We will show that if a set is self-similar, as in the definition above, then it is, in fact, the intersection of countably many sets.

Lemma 2 If $\left\{f_{k}\right\}_{k=1}^{n}$ is a family of contractive similitudes defined on $\mathbb{R}^{n}$, then there is a compact subset $B$ of $\mathbb{R}^{n}$ satisfying $f_{k}(B) \subseteq B$ for all $k$.

\section{Proof:}

Let $r$ be the maximum distance from the origin of the images of the origin under the family of maps $\left\{f_{k}\right\}$. Let $c$ be the maximum of the contraction ratios of the $f_{k}$ 's. Let $B$ be aclosed ball centered at the origin of radius $\frac{r}{1-c}$. For any $x$ in this ball and any $f_{k}$, we have $\left|f_{k}(x)\right| \leq$ $r+c_{k} \frac{r}{1-c} \leq r+c \frac{r}{1-c}=\frac{r}{1-c}$.

Let $\left\{S_{k}\right\}_{k=1}^{n}$ be a family of contractive similitudes on $\mathbb{R}^{n}$, and let $D$ be a compact set satisfying, for all $k, S(D) \subseteq D$. Define $F(D)$ to be $\cup_{k=1}^{n} S_{k}(D)$ and define $F^{k}(D)$ to be the iterates of $D$ under $F: F^{0}(D)=D$ and $F^{n}(D)=F\left(F^{n-1}(D)\right)$ for $n \geq 1$. 
Proposition 4 [4] Let $\left\{f_{k}\right\}_{k=1}^{n}$ be a family of contractive similitudes on $\mathbb{R}^{n}$ and let $D$ be a compact set satisfying, for all $k, F(D) \subseteq D$. Then $E=\cap_{n=1}^{\infty} F^{n}(D)$ is a nonempty invariant set for $\left\{f_{k}\right\}$, in the sense of definition 9 , and $E$ is the only set satisfying the definition of invariant set for $\left\{f_{k}\right\}$.

\section{Proof:}

We will follow the proof given in [4]. Let $E=\cup_{1}^{m} S_{i}(E)$, where $S_{i}$ are contractive similitudes, with similitude ratios $c_{i}$. Note, by definition $E$ is compact and is an invariant set. Let $D$ and $F(D)$ be as given. By Lemma $2, S^{k}(D)=S^{k-1}(S(D)) \subseteq S^{k-1}(D)$. Thus, we have that $\left\{S^{k}(D)\right\}$ is a nested sequence of nonempty compact sets. This gives us that $G=\cap_{1}^{\infty} S^{k}(D) \neq$ $\emptyset$. We also have that $S(G)=G$ is an invariant set. If we can show that this invariant set is unique, then we have $E=G$.

Let $A$ and $B$ be compact sets. Then

$d(S(A), S(B))=d\left(\cup_{1}^{m} S_{i}(A), \cup_{1}^{m} S_{i}(B)\right) \leq \max _{1 \leq i \leq m} d\left(S_{i}(A), S_{i}(B)\right) \leq\left(\max _{1 \leq i \leq m} c_{i}\right) d(A, B)$.

If $S(A)=A$ and $S(B)=B$ then $A=B$ because only $d(A, B)=0$ satisfies $d(A, B) \leq$ $\max _{1 \leq i \leq m} c_{i} d(A, B)$.

We know $S(E)=E$ and $S(G)=G$. We also have $E$ and $G$ are compact. Thus, we have that $E$ and $G$ are equal invariant sets. Thus $\cap_{1}^{\infty} S^{k}(D)=E=\cup_{1}^{m} S_{i}(E)$. $\square[4]$

Note that with existence and uniqueness established, it is often useful to use $B=E$ in proposition 4, i.e. to write $E=\cap_{n=1}^{\infty} F^{n}(E)$. 
Let us look at a few different examples of self-similar sets. We will show these sets are self-similar using definition 9. First, is what is known as the Sierpinski Carpet. Take the closed unit square in $\mathbb{R}$, that is $[0,1] \times[0,1]$. Break the square into 9 equal subsquares, as pictured below.

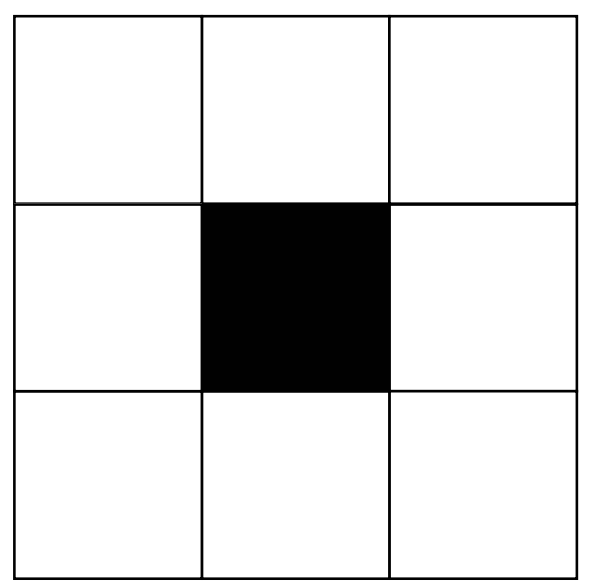

Figure 5.1: First Stage in Sierpinski Carpet Formation

Now remove the open middle square from the second row. Break up each of the eight remaining squares into nine equal subsquares as above. Again, remove the middle square. Repeat this process indefinitely. This will give you what is called the Sierpinski Carpet. The Sierpinski Carpet is self-similar. Let $f_{1}(x, y)=\left(\frac{1}{9} x, \frac{1}{9} y\right), f_{2}(x, y)=\left(\frac{1}{9} x+\frac{1}{3}, \frac{1}{9} y\right), f_{3}(x, y)=$ $\left(\frac{1}{9} x+\frac{2}{3}, \frac{1}{9} y\right), f_{4}(x, y)=\left(\frac{1}{9} x, \frac{1}{9} y+\frac{1}{3}\right), f_{5}(x, y)=\left(\frac{1}{9} x+\frac{1}{3}, \frac{1}{9} y+\frac{1}{3}\right), f_{6}(x, y)=\left(\frac{1}{9} x, \frac{1}{9} y+\frac{2}{3}\right)$, $f_{7}(x, y)=\left(\frac{1}{9} x+\frac{1}{3}, \frac{1}{9} y+\frac{2}{3}\right)$ and $f_{8}(x, y)=\left(\frac{1}{9}+\frac{2}{3}, \frac{1}{9} y+\frac{2}{3}\right)$. Each of these are clearly contractive similitudes with ratios $\frac{1}{9}$. Also $S=\cup_{1}^{8} f_{i}(S)$. Hence, the Sierpinski Carpet is a self-similar set. 
Proposition 5 The Sierpinski Carpet has Hausdorff dimension $\frac{\ln (8)}{\ln (3)}$.

\section{Proof:}

Let $S$ denote the Sierpinski Carpet.

First, we will show the Hausdorff dimension of $S$ is less than or equal to $\frac{\ln (8)}{\ln (3)}$.

A closed ball of radius $3^{-j} \frac{\sqrt{2}}{2}$ covers the entire level- $j$ square of $S$ for which $a_{j}$ is the center.

Thus,

$H^{s}(S) \leq \lim _{j \rightarrow \infty} 8^{j}(\sqrt{2})^{s} 3^{-s j}$.

Hence,

$H^{s}(S) \leq(\sqrt{2})^{s} l i m_{j \rightarrow \infty} 8^{j} 3^{-s j}$.

If $s \geq \frac{\ln (8)}{\ln (3)}$ then $3^{-s} 8 \leq 1$ and so $\ln \left(3^{-s} 8\right) \leq 0$.

This gives that $\lim _{j \rightarrow \infty} j \ln \left(3^{-s} 8\right) \leq 0$. So $\lim _{j \rightarrow \infty} 3^{-s j} 8^{j} \leq 1$ and thus $H^{s}(S)<\infty$.

Now, we will show that the Hausdorff dimension of $S$ is greater than or equal to $\frac{\ln (8)}{\ln (3)}$ by showing $H^{\frac{\ln (8)}{\ln (3)}}(S)>0$.

We begin as in the proof of the analogous statement for the square.

Take $\delta>0$. Cover $S$ by arbitrary sets of diameter less than $\delta$. Note that we need to cover $S$, not the entire unit square. However, every one of the $8^{k}$ interiors of the standard squares with side length $\frac{1}{3^{k}}$ includes a point from $S$, so covers have to hit the interior of every one of those squares. The sets might as well be closed balls, call them $\left\{B_{j}\right\}$. For each ball $B_{j}$ 
there exists $k_{j}$ so that $\frac{1}{3^{k_{j}+1}} \leq \operatorname{diam}\left(B_{j}\right)<\frac{1}{3^{k_{j}}}$.

Choose $\epsilon>0$. Expand $B_{j}$ to a closed ball $B_{j}^{\prime}$ so that $\operatorname{diam}\left(B_{j}^{\prime}\right)$ satisfies $\frac{1}{3^{k_{j}+1}} \leq \operatorname{diam}\left(B_{j}^{\prime}\right)<$ $\frac{1}{3^{k_{j}}}$ and so that $\left(\operatorname{diam}\left(B_{j}^{\prime}\right)\right)^{\frac{\ln (8)}{\ln (3)}}-\left(\operatorname{diam}\left(B_{j}\right)\right)^{\frac{\ln (8)}{\ln (3)}} \leq \frac{\epsilon}{2^{j}}$. This is possibly by the continuity of powers.

The interiors of the $B_{j}^{\prime}$ 's form an open cover of $S$ and thus by compactness we may choose a finite subcover and return to closed balls. Let $\left\{B_{j_{1}}^{\prime}, \ldots B_{j_{n}}^{\prime}\right\}$ denote the collection of closed balls.

Let $k_{0}=\max _{j_{1}, \ldots, j_{n}} k_{j}$ which were chosen above. Note that if $\frac{1}{3^{k+1}} \leq \operatorname{diam}\left(B_{j}\right)<\frac{1}{3^{k}}$ then $B_{j}^{\prime}$ can intersect a most four of the interiors of the squares in the standard decomposition into squares of side length $\frac{1}{3^{k}}$. The same $B_{j}^{\prime}$ 's can intersect at most $4 \cdot 8^{k_{0}-k}$ of the interiors of squares in the standard decomposition of our given square into squares of side length $\frac{1}{3^{k_{0}}}$. Note,

$$
\begin{gathered}
4 \cdot 8^{\left(k_{0}-k\right)}=4 \cdot 8^{k_{0}} 8^{-k}=4 \cdot 8^{k_{0}} \cdot \frac{1}{\left(3^{k}\right)^{\frac{\ln (8)}{\ln (3)}}} \\
=4 \cdot 8^{k_{0}} \cdot 3^{\frac{\ln (8)}{\ln (3)}} \cdot \frac{1}{\left(3^{k+1}\right)^{\frac{\ln (8)}{\ln (3)}}} \leq 4 \cdot 8^{k_{0}} \cdot 3^{\frac{\ln (8)}{\ln (3)}}\left(\operatorname{diam}\left(B_{j}^{\prime}\right)\right)^{\frac{\ln (8)}{\ln (3)}} .
\end{gathered}
$$

To hit all $8^{k_{0}}$ interiors we need

$$
8^{k_{0}} \leq \Sigma_{j} 4 \cdot 8^{k_{0}} 3^{\frac{\ln (8)}{\ln (3)}}\left(\operatorname{diam}\left(B_{j}^{\prime}\right)\right)^{\frac{\ln (8)}{\ln (3)}}
$$


So,

$$
\frac{1}{4 \cdot 3^{\frac{\ln (8)}{\ln (3)}}} \leq\left(\operatorname{diam}\left(B_{j}^{\prime}\right)\right)^{\frac{\ln (8)}{\ln (3)}}
$$

Because

$$
\left(\operatorname{diam}\left(B_{j}^{\prime}\right)\right)^{\frac{\ln (8)}{\ln (3)}} \leq\left(\operatorname{diam}\left(B_{j}\right)\right)^{\frac{\ln (8)}{\ln (3)}}+\epsilon .
$$

and because $\epsilon$ was arbitrary, we have that

we have that $H^{\frac{\ln (8)}{\ln (3)}}(S)>\frac{1}{4 \cdot 3^{\frac{\ln (8)}{\ln (3)}}}>0$.

Next let us form what is known as the Sierpinski Gasket or the Sierpinski Triangle. Begin with an equilateral triangle. Break the triangle into four equal-sized equilateral triangles. Remove the middle triangle and keep the three triangles in the corners. Now break up the three smaller triangles and continue this process indefinitely. A similar proof as above will show the dimension of the Sierpinski Triangle is $\frac{\ln (3)}{\ln (2)}$.

Lastly, let us look at an example of a fractal tree. Start off with $[0,1] \times[0,1]$ and as in the Sierpinski Carpet, break this set into nine equal subsquares. Keep only the square in the center and those in the corners. Repeating this process gives what is known as a fractal tree. This particular tree has dimension $\frac{\ln (5)}{\ln (3)}$. This is proven in a similar manner as the dimension of the Sierpinski Carpet. There are many different examples of fractal trees. Instead of removing the 4 squares we chose above one could remove only those square in the corners, 
etc.

We need not only look at subsets of $\mathbb{R}^{2}$. What if we looked at the unit cube, a subset of $\mathbb{R}^{3}$ ? Break this cube into 27 subcubes of equal size, i.e. with height $\frac{1}{3}$. To begin, remove all but the 8 cubes in the corners. At the next stage remove all but the 8 subcubes in the corners of each remaining cube. Repeat this process indefinitely. We then get a fractal tree; call it $T$. We will find a surprising result after calculating the dimension of $T$. We will find that the dimension of $T$ is the same as the dimension of the Sierpinski carpet.

Proposition 6 The Hausdorff dimension of $T$ is $\frac{\ln (8)}{\ln (3)}$.

\section{Proof:}

First, we will show the Hausdorff dimension of $T$ is less than or equal to $\frac{\ln (8)}{\ln (3)}$.

A closed ball of radius $3^{-j} \frac{\sqrt{3}}{2}$ covers the entire level- $j$ cube of $T$ for which $a_{j}$ is the center.

Thus,

$H^{s}(T) \leq \lim _{j \rightarrow \infty} 8^{j}(\sqrt{3})^{s} 3^{-s j}$.

Hence,

$H^{s}(T) \leq(\sqrt{3})^{s} \lim _{j \rightarrow \infty} 8^{j} 3^{-s j}$

If $s \geq \frac{\ln (8)}{\ln (3)}$ then $3^{-s} 8 \leq 1$ and so $\ln \left(3^{-s} 8\right) \leq 0$.

This gives that $\lim _{j \rightarrow \infty} j \ln \left(3^{-s} 8\right) \leq 0$. So $\lim _{j \rightarrow \infty} 3^{-s j} 8^{j} \leq 1$ and thus $H^{s}(T)<\infty$.

Now, we will show that the Hausdorff dimension of $T$ is greater than or equal to $\frac{\ln (8)}{\ln (3)}$ by 
showing $H^{\frac{\ln (8)}{\ln (3)}}(T)>0$.

We begin as in the proof of the analogous statement for the carpet.

Take $\delta>0$. Cover $T$ by arbitrary sets of diameter less than $\delta$. Note that we need to cover $T$, not the entire unit cube. However, every one of the $8^{k}$ interiors of the standard cubes with side length $\frac{1}{3^{k}}$ includes a point from $T$, so covers have to hit the interior of every one of those cubes. Thus the sets might as well be closed balls, call them $\left\{B_{j}\right\}$. For each ball $B_{j}$ there exists $k_{j}$ sjo that $\frac{1}{3^{k_{j}+1}} \leq \operatorname{diam}\left(B_{j}\right)<\frac{1}{3^{k_{j}}}$.

Choose $\epsilon>0$. Expand $B_{j}$ to a closed ball $B_{j}^{\prime}$ so that $\operatorname{diam}\left(B_{j}^{\prime}\right)$ satisfies $\frac{1}{3^{k_{j}+1}} \leq \operatorname{diam}\left(B_{j}^{\prime}\right)<$ $\frac{1}{3^{k_{j}}}$ and so that $\left(\operatorname{diam}\left(B_{j}^{\prime}\right)\right)^{\frac{\ln (8)}{\ln (3)}}-\left(\operatorname{diam}\left(B_{j}\right)\right)^{\frac{\ln (8)}{\ln (3)}} \leq \frac{\epsilon}{2^{j}}$. This is possibly by the continuity of powers.

The interiors of the $B_{j}^{\prime}$ 's form an open cover of $T$ and thus by compactness we may choose a finite subcover and return to closed balls. Let $\left\{B_{j_{1}}^{\prime}, \ldots B_{j_{n}}^{\prime}\right\}$ denote the collection of closed balls.

Let $k_{0}=\max _{j_{1}, \ldots, j_{n}} k_{j}$ which were chosen above. Note that if $\frac{1}{3^{k+1}} \leq \operatorname{diam}\left(B_{j}\right)<\frac{1}{3^{k}}$ then $B_{j}^{\prime}$ can intersect a most eight of the cubes in the standard decomposition into cubes of side length $\frac{1}{3^{k}}$. The same $B_{j}^{\prime}$ 's can intersect at most $8 \cdot 8^{k_{0}-k_{j}}$ of the interiors of cubes in the standard decomposition of our given cube into cubes of side length $\frac{1}{3^{k_{0}}}$. Note,

$$
8 \cdot 8^{\left(k_{0}-k_{j}\right)}=8 \cdot 8^{k_{0}} 8^{-k_{j}}=8 \cdot 8^{k_{0}} \cdot \frac{1}{\left(3^{k_{j}}\right)^{\frac{\ln (8)}{\ln (3)}}}
$$




$$
=8 \cdot 8^{k_{0}} \cdot 3^{\frac{\ln (8)}{\ln (3)}} \cdot \frac{1}{\left(3^{k+1}\right)^{\frac{\ln (8)}{\ln (3)}}} \leq 8 \cdot 8^{k_{0}} \cdot 3^{\frac{\ln (8)}{\ln (3)}}\left(\operatorname{diam}\left(B_{j}^{\prime}\right)\right)^{\frac{\ln (8)}{\ln (3)}}
$$

To hit all $8^{k_{0}}$ interiors we need

$$
8^{k_{0}} \leq \Sigma_{j} 8 \cdot 8^{k_{0}} 3^{\frac{\ln (8)}{\ln (3)}}\left(\operatorname{diam}\left(B_{j}^{\prime}\right)\right)^{\frac{\ln (8)}{\ln (3)}}
$$

So,

$$
\frac{1}{8 \cdot 3^{\frac{\ln (8)}{\ln (3)}}} \leq\left(\operatorname{diam}\left(B_{j}^{\prime}\right)\right)^{\frac{\ln (8)}{\ln (3)}}
$$

Because

$$
\left(\operatorname{diam}\left(B_{j}^{\prime}\right)\right)^{\frac{\ln (8)}{\ln (3)}} \leq\left(\operatorname{diam}\left(B_{j}\right)\right)^{\frac{\ln (8)}{\ln (3)}}+\epsilon .
$$

and because $\epsilon$ was arbitrary, we have that

we have that $H^{\frac{\ln (8)}{\ln (3)}}(T)>\frac{1}{8 \cdot 3^{\frac{\ln (8)}{\ln (3)}}}>0$.

We have now shown that $T$ has the same dimension as the Sierpinski carpet. However, the carpet has many different rectifiable paths, such as the path from $(0,0)$ to $(0,1)$, the path from $(0,1)$ to $(1,1)$, as well as the path from $(1,1)$ to $(1,0)$, and many others. We omit the proof that the fractal tree, with retracing ruled out, has only a unique path connecting two distinct points. Hence they cannot be bilipschitz equivalent. 
Now, let us move back to Cantor sets. There is another standard construction of the middle third Cantor set. It turns out that the Cantor set discussed earlier is simply all points in the interval $[0,1]$ that can be written in at least one way in base three just using the digits 0 and 2. However, there is at least one more construction of the classical Cantor Set which leads to the construction of infinitely more Cantor Sets of different dimensions.

Definition 11 Let $F=\{0,1, \ldots, n-1\}$. Let $F^{\infty}$ denote the set of all sequences of elements of $F$. Let $x=x_{1} x_{2} x_{3} \ldots, y=y_{1} y_{2} y_{3} \ldots \in F^{\infty}$, and define $L(x, y)=n$ to mean $x_{i}=y_{i}$ for $1 \leq i \leq n$ and $x_{n+1} \neq y_{n+1}$. Let $0<a<1$ and define $d_{a}(x, y)=a^{L(x, y)}$ with $a^{\infty}=0$. Then $\left\{F^{\infty}, d_{a}\right\}$ is a metric space and called a Cantor Set. [1]

Proposition $7\left\{F^{\infty}, d_{a}\right\}$ is an ultrametric space and has no rectifiable paths.

\section{Proof:}

Let $\left\{F^{\infty}, d_{a}\right\}$ be a Cantor set. Take $x, y, z \in F^{\infty}$. Then $L(x, z) \geq \min \{L(x, y), L(y, z)\}$ and hence $d_{a}(x, z) \leq \max \left\{d_{a}(x, y), d_{a}(y, z)\right\}$. Now, we do in fact have an ultrametric space. Since we have shown that ultrametric spaces have no rectifiable paths we know that there are no rectifiable paths in $\left\{F^{\infty}, d_{a}\right\}$. 
Proposition $8[1]$

The Hausdorff dimension of $\left(F^{\infty}, d_{a}\right)$ for $|F|=k$ is $\frac{\ln (k)}{\ln \left(\frac{1}{a}\right)}$.

\section{Proof:}

First, we will show the Hausdorff dimension is less than or equal to $\frac{\ln \left(\frac{1}{k}\right)}{\ln (a)}=\frac{\ln (k)}{\ln \left(\frac{1}{a}\right)}$.

For any $n$ we can cover with $k^{n}$ balls of diameter $2 a^{n}$. So if we have that $d \geq \frac{\ln \left(\frac{1}{k}\right)}{\ln (a)}$ then

$H^{d}\left(F^{\infty}\right) \leq k^{n}\left(2 a^{n}\right)^{d} \leq 2^{\frac{\ln \left(\frac{1}{k}\right)}{\ln (a)}} \lim _{n \rightarrow \infty} \frac{k^{n}}{k^{n}}=2^{\frac{\ln \left(\frac{1}{k}\right)}{\ln (a)}}<\infty$

Take $\delta>0$. Cover $F^{\infty}$ by arbitrary sets of diameter less than $\delta$. Similarly as the square and carpet arguments, at level $m$ we have $k^{m}$ basic sets, each of distance at least $a^{m-1}$ from each other. So the sets might as well be closed balls, call them $\left\{B_{j}\right\}$. For each ball $B_{j}$ there exists $m_{j}$ so that $a^{m_{j}+1} \leq \operatorname{diam}\left(B_{j}\right)<a^{m_{j}}$.

Choose $\epsilon>0$. Expand $B_{j}$ to a closed ball $B_{j}^{\prime}$ so that $\operatorname{diam}\left(B_{j}^{\prime}\right)$ satisfies $a^{m_{j}+1} \leq \operatorname{diam}\left(B_{j}^{\prime}\right)<$ $a^{m_{j}}$ and so that $\left(\operatorname{diam}\left(B_{j}^{\prime}\right)\right)^{\frac{\ln (k)}{\ln \left(\frac{1}{a}\right)}}-\left(\operatorname{diam}\left(B_{j}\right)\right)^{\frac{\ln (k)}{\ln \left(\frac{1}{a}\right)}} \leq \frac{\epsilon}{2^{j}}$. This is possibly by the continuity of powers.

The interiors of the $B_{j}^{\prime}$ 's form an open cover of $F^{\infty}$ and thus by compactness we may choose a finite subcover and return to closed balls. Let $\left\{B_{j_{1}}^{\prime}, \ldots B_{j_{n}}^{\prime}\right\}$ denote the collection of closed balls.

Let $k_{0}=\max _{j_{1}, \ldots, j_{n}} k_{j}$ which were chosen above. Note that if $a^{m+1} \leq \operatorname{diam}\left(B_{j}^{\prime}\right)<a^{m}$ then $B_{j}^{\prime}$ 's can intersect at most one of the interiors of sets at level $m$ and so can hit at most $k^{m_{0}-m_{j}}$ of sets at level $m_{0}$. 
Thus,

$$
\begin{aligned}
& k^{m_{0}-m}=k^{m_{0}} k^{-m}=k^{m_{0}}\left(a^{m}\right)^{\frac{\ln (k)}{\ln (a)}} \\
& =k^{m_{0}} a^{\frac{\ln (k)}{\ln (a)}}\left(a^{-\left(m_{j}+1\right)}\right)^{\frac{\ln \left(\frac{1}{k}\right)}{\ln (a)}} \\
& \leq k^{m_{0}} k\left(\operatorname{diam}\left(B_{j}^{\prime}\right)\right)^{\frac{\ln \left(\frac{1}{k}\right)}{\ln (a)}}
\end{aligned}
$$

So,

$k^{m_{0}} \leq \Sigma_{j} k^{m_{0}} k\left(\operatorname{diam}\left(B_{j}^{\prime}\right)^{\frac{\ln \left(\frac{1}{k}\right)}{\ln (a)}}\right.$

and thus

$$
\frac{1}{k} \leq \Sigma_{j} k^{m_{0}}\left(\operatorname{diam}\left(B_{j}^{\prime}\right)^{\frac{\ln \left(\frac{1}{k}\right)}{\ln (a)}} \leq \Sigma_{j} k^{m_{0}}\left(\operatorname{diam}\left(B_{j}\right)^{\frac{\ln \left(\frac{1}{k}\right)}{\ln (a)}}+\epsilon\right.\right.
$$

Thus $H^{\frac{\ln \left(\frac{1}{k}\right)}{\ln (a)}}\left(F^{\infty}\right)>0$ and we have that the Hausdorff dimension of $F^{\infty}$ is $\frac{\ln (k)}{\ln \left(\frac{1}{a}\right)}$ as desired.

Example $8\left(F^{\infty}, d_{a}\right)$ with $|F|=8$ and $a=\frac{1}{3}$ has Hausdorff dimension $\frac{\ln (8)}{\ln (3)}$.

We have shown that ultrametric spaces have no nonconstant paths, and hence they have no paths of positive length. By the previous proposition the dimension of $\left(F, d_{a}\right)$ is $\frac{\ln (8)}{\ln (3)}$, as desired.

Thus, we have that $S, T$ and $\left(F, d_{a}\right)$ have the same Hausdorff dimension. Now, $S$ has multiple paths connecting two distinct points, $T$ has a unique path, up to retracing, connecting two distinct points and $\left(F, d_{a}\right)$ has no nonconstant rectifiable paths.

Thus, having the same Hausdorff dimension does not guarantee bilipschitz equivalence. The 
Sierpinski Carpet and Triangle, as well as fractal trees and Cantor sets, are all examples of self-similar sets. 


\section{Chapter 6}

\section{Bilipschitz Equivalence of Cantor Sets}

As a way to visualize Cantor sets of non-integer dimension, we identify a bilipschitz equivalence between each such Cantor set constructed using $|F|=2$ and a corresponding subset of Euclidean space. The argument does not extend to Cantor sets constructed from $|F|=2$ that have integer dimension, although in those spaces our construction does define a Lipschitz surjection onto a Cartesian product of unit intervals.

Theorem 1 Let $|F|=2 .\left(F^{\infty}, d_{a}\right)$ is bilipschitz equivalent to a subset of $[0,1]$ when $0<$ $a<\frac{1}{2}$. When $\frac{1}{\sqrt[n-1]{2}}<a<\frac{1}{\sqrt[n]{2}}$ with $n>1$ then $\left(F^{\infty}, d_{a}\right)$ is bilipschitz equivalent to a subset of $[0,1] \times \ldots \times[0,1]=[0,1]^{n}$. 


\section{Proof:}

Let us look at the case where $0<a<\frac{1}{2}$ and $|F|=2$. We will first construct the subset of $[0,1]$ to which $F^{\infty}$ is equivalent. Call this set $C_{a}$. We will define this set inductively. For the first step remove the open middle interval of $[0,1]$ of length $1-2 a$. Then for step $n$ for each of the $2^{n}$ intervals remove the open middle interval of length $a^{n-1}-2 a^{n}$. This gives us $2^{n+1}$ intervals remaining of length $a^{n}$. Notice that this corresponds directly with the known inductive procedure for constructing the classical Cantor set when $a=\frac{1}{3}$.

Now let us construct the map. We will again define this map inductively. Let $x=$ $x_{1} x_{2} x_{3} \ldots \in F^{\infty}$. If $x_{1}=0$ then $f(x) \in[0, a]$, the set on the left after the removal of the interval in step one of the creation of $C_{a}$. If $x_{1}=1$ then $f(x) \in[1-2 a, 1]$, the set of the right after the removal of the interval in step one of creating $C_{a}$. Now, for the $n^{\text {th }}$ step of this process, we have that $f(x)$ is in an interval of length $a^{n-1}$ created in the $n-1^{s t}$ step of the $C_{a}$ formation. Let this set be denoted by $\left[y, y+a^{n-1}\right]$. If $x_{n}=0$ then $f(x) \in\left[y, y+a^{n}\right]$, the set of the left in the $n^{t h}$ step of the $C_{a}$ formation. However, if $x_{n}=1$ then $f(x) \in\left[y+a^{n-1}-a^{n}, y+a^{n-1}\right]$, the set on the right. By construction we have a one to one map onto $C_{a}$.

Let us show that $f$ is a bilipschitz map. Let $x, y \in F^{\infty}$ with $L(x, y)=n$ defined previously as the number of integer places that $x_{i}=y_{i}$ from $1 \leq i \leq n$. Then, we have from definition of $f$ that $f(x), f(y)$ are in the same subinterval of length $a^{n}$ so $d(f(x), f(y)) \leq a^{n}$. Now $f(x), f(y)$ are in different subintervals of length $a^{n+1}$ which are of length $a^{n}-2 a^{n+1}$ apart. Thus, we have that $d(f(x), f(y)) \geq a^{n}-2 a^{n+1}$. Choose $K=\frac{1}{1-2 a}$. Note $K$ is defined since 
$0<a<\frac{1}{2}$. Now

$$
K^{-1} d_{a}(x, y)=K^{-1} a^{n}=(1-2 a) a^{n}=a^{n}-2 a^{n+1} \leq d(f(x), f(y))
$$

and

$$
d(f(x), f(y)) \leq a^{n} \leq \frac{1}{1-2 a} a^{n}=K a^{n}=K d_{a}(x, y)
$$

Thus, we have that

$$
K^{-1} d_{a}(x, y) \leq d(f(x), f(y)) \leq K d_{a}(x, y)
$$

and hence $f$ is a bilipschitz map of $\left(F^{\infty}, d_{a}\right)$ onto $\left(C_{a}, d\right)$ as desired.

Now, let us look at the case with $\frac{1}{\sqrt[n-1]{2}}<a<\frac{1}{\sqrt[n]{2}}$ with $n>1$ and $|F|=2$. Again, let us first construct $C_{a}$. For each copy of $[0,1]$ form $C_{a^{n}}$. Then $C_{a}=C_{a^{n}}^{n}=C_{a^{n}} \times C_{a^{n}} \times \ldots \times C_{a^{n}}$, i.e. the product of $n$ copies of $C_{a^{n}}$.

Now form $f: F^{\infty} \rightarrow C_{a}$. Let $x=x_{11} x_{12} \ldots x_{1 n} x_{21} x_{22} \ldots x_{2 n} x_{31} \ldots \in F^{\infty}$. Look at the sequence $x_{1 j} x_{2 j} x_{3 j} \ldots$ for each $1 \leq j \leq n$. For the $j^{t h} C_{a^{n}}$ in the formation of $C_{a}$ send $x_{1 j} x_{2 j} x_{3 j} \ldots$ into $C_{a^{n}}$ as the previous case when $0<a<\frac{1}{2}$. Do this for each $j$ with $1 \leq j \leq n$. Thus we have a one to one map of $\left(F^{\infty}, d_{a}\right)$ onto $\left(C_{a}, d\right)$. It turns out that this map is bilipschitz. 
To show that this map $f: F^{\infty} \rightarrow C_{a}$ is bilipschitz, take $x, y \in F^{\infty}$. Let $L(x, y)=m n+r-1$ where $1 \leq r \leq n$. By construction $f(x)$ and $f(y)$ are in the same $n$-dimensional cube of side length $a^{m n}$. Thus $d(f(x), f(y)) \leq \sqrt{n} a^{m n}$. Now, $f(x)$ and $f(y)$ are in different $n$ dimensional cubes of side length $a^{(m+1) n}$. So $d(f(x), f(y)) \geq a^{m n}-2 a^{(m+1) n}=a^{m n}(1-2 a)^{n}$. Choose $K=\max \left\{\frac{1}{1-2 a}^{n}, a^{-n} \sqrt{n}\right\}$ and note $1-2 a<1$. Then we see that

$$
K^{-1} d_{a}(x, y)=K^{-1} a^{m n+r-1} \leq(1-2 a)^{n} a^{m n+r-1} \leq a^{m n}-2 a^{(m+1) n} \leq d(f(x), f(y))
$$

Also,

$$
d(f(x), f(y)) \leq \sqrt{n} a^{m n} \leq \sqrt{n} a^{-n} a^{m n+n} \leq K a^{m n+n} \leq K a^{m n+r-1}=K d_{a}(x, y)
$$

Thus we have that $f$ is a bilipschitz map and hence $\left(F^{\infty}, d_{a}\right)$ is bilipschitz equivalent to $\left(C_{a}, d\right)$, a subset of $([0,1], d)$ as desired.

Now, what if we looked at the maps for $a=\frac{1}{\sqrt[n]{2}}$. Then our map would not be one to one. For example, if $a=\frac{1}{2}$ both $1,0,0,0, \ldots$ and $0,1,1,1,1, \ldots$ would be mapped to the point $\frac{1}{2}$. Thus, these maps are not one to one; however, bilipschitz maps are one to one. Thus, our map is not a bilipschitz map of $\left(F, d_{a}\right)$ onto $\left([0,1]^{n}, d\right)$.

We will now state this fact in a theorem and give an alternate proof. 
Theorem 2 When $a=\frac{1}{\sqrt[n]{2}}$ with $n \in \mathbb{N}$ and $|F|=2$ then $\left(F^{\infty}, d_{a}\right)$ is not bilipschitz equivalent to $[0,1]^{n}$. However, we still have $d(f(x), f(y)) \leq K d(x, y)$ even if $f(x)=f(y)$. Clearly, our map is still surjective. Hence, we have a Lipschitz map which is surjective.

\section{Proof:}

Let $a=\frac{1}{\sqrt[n]{2}}$ with $n \in \mathbb{N}$. Let $|F|=2$. As shown previously, $\left(F^{\infty}, d_{a}\right)$ is an ultrametric space. Thus, we know that it has no rectifiable paths, unlike its counterpart $[0,1]^{n}$. The presence of rectifiable paths are preserved under bilipschitz equivalence, and hence the two spaces cannot be bilipschitz equivalent. 


\section{Chapter 7}

\section{A Surprising Equivalence}

Now, we will move on to discuss a surprising result of H. Rao, H. J. Ruan and L. F. Xi [2]. G. David and S. Semmes, [1], first asked in their 1997 book Fractured Fractals and Broken Dreams whether there is a bilipschitz equivalence of $M$ and $M^{\prime}$, two compact self-similar sets in $\mathbb{R}$, where $M$ is given by the iterated function scheme $\left\{\phi_{1}(x)=\frac{1}{5} x, \phi_{2}(x)=\frac{1}{5} x+\frac{2}{5}, \phi_{3}(x)=\right.$ $\left.\frac{1}{5} x+\frac{4}{5}\right\}$ and $M^{\prime}$ is given by $\left\{\psi_{1}(x)=\frac{1}{5} x, \psi_{2}(x)=\frac{1}{5} x+\frac{3}{5}, \psi_{3}(x)=\frac{1}{5} x+\frac{4}{5}\right\}$. Rao, Ruan, and $\mathrm{Xi}$ showed that there is a bilipschitz map between these sets. When pictured, these sets do not look like they are bilipschitz equivalent, so this is a very surprising bilipschitz equivalence. To prove this result one must first delve into some graph theory as well as recall some terms.

Recall the following definitions:

A mapping $f: \mathbb{R}^{n} \rightarrow \mathbb{R}^{n}$ is a contractive similitude if there exists $r<1$ for all $x, y \in \mathbb{R}^{n}$

then $d(f(x), f(y))=r d(x, y)$, where $d$ is the Euclidean metric. The number $r$ is called 
contraction ratio or similitude ratio [2].

Let $\left\{f_{k}\right\}_{k=1}^{N}$, mapping $\mathbb{R}^{n}$ into itself, be a family of contractive similitudes. Let $\mathrm{E}$ be a nonempty compact set, where $E=\cup_{k=1}^{N} f_{k}(E)$. Then the set $E$ is called the invariant set or the self-similar set of $\left\{f_{k}\right\}_{k=1}^{N}$. If $f_{i}(E) \cap f_{j}(E)=\emptyset$ for all $i \neq j$ then $E$ is called dust-like [2].

Let $\left\{f_{k}\right\}_{k=1}^{N}$, mapping $\mathbb{R}^{n}$ into itself, be a family of contractive similitudes. Let $\mathrm{E}$ be a nonempty compact set, where $E=\cup_{k=1}^{N} f_{k}(E)$. Then the set $E$ is called the invariant set or the self-similar set of $\left\{f_{k}\right\}_{k=1}^{N}$. If $f_{i}(E) \cap f_{j}(E)=\emptyset$ for all $i \neq j$ then $E$ is called dust-like [2].

Now, to begin our discussion let $\left\{f_{k}\right\}_{k=1}^{N}$ and $\left\{g_{k}\right\}_{k=1}^{N}$, mapping $\mathbb{R}^{n}$ into itself, be two families of contractive similitudes. Let $E$ and $F$ be the respective invariant sets for $\left\{f_{k}\right\}_{k=1}^{N}$ and $\left\{g_{k}\right\}_{k=1}^{N}$. Assume $E$ and $F$ are both dust-like. Assume for all $k$, the contraction ratio for $f_{k}$ is the same as contraction ratio for $g_{k}$. It will be shown that $E$ is bilipschitz equivalent to $F$.

We want to find invariant sets that coincide with what is called a graph directed set. To do this we will first define $G=(V, \Gamma)$ to be a directed graph where $V=\{1, \ldots, N\}$ denotes the set of vertices and $\Gamma$ denote the edges and their direction. We also assume that each vertex $v$ has at least one edge beginning at $v$ itself. Also, we will assume that for all $e \in \Gamma$ there exists a contractive similitude, denoted $T_{e}$, that maps $\mathbb{R}^{n}$ to $\mathbb{R}^{n}$ with similarity ratio, denoted $\rho_{e}$, in $(0,1)$. Let $\Gamma_{i, j}$ denote the set of all edges beginning at vertex $i$ and ending at 
vertex $j$. [2] We need two more definitions before we discuss a few examples.

Definition 12 [2] A labeled directed graph, denoted $G^{*}$, is a graph $G=(V, \Gamma)$ with similitudes $T_{e}: \mathbb{R}^{n} \rightarrow \mathbb{R}^{n}$ for all $e \in \Gamma$.

Definition 13 [2] $A$ graph directed set on $G^{*}$ is a set of compact nonempty sets $E_{i}$ that satisfy:

$$
E_{i}=\cup_{j=1}^{N} \cup_{e \in \Gamma_{i, j}} T_{e}\left(E_{j}\right)
$$

$\left\{E_{i}\right\}$ is said to be dust-like if, for each $i$, the above union is disjoint.

One can show that, for a given $G^{*}$, there is a unique graph-directed set. Let us look at an example of a graph directed set.

Example 9 The graph directed set for the standard middle thirds Cantor set consists of a single vertex and two directed edges

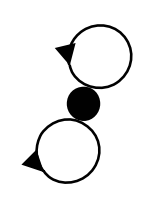

Figure 7.1: Directed Graph for Cantor Set

Each point in the middle thirds Cantor set encodes a trip covering countably many edges (this graph, and every such trip is encoded by a point in the Cantor set.) At the nth stage 
of your travel you can either go through the path $e_{1}$ or $e_{2}$. If the nth digit is a zero travel through the path $e_{1}$, if the nth digit is a two travel through the path $e_{2}$.

Now let us define another property of graph directed sets. This will help us in our goal of showing that $M$ and $M^{\prime}$, as described in the chapter introduction, are bilipschitz equivalent.

Theorem 3 [2] Let $G^{*}$ and $H^{*}$ be labeled directed graphs. Let $\left\{E_{i}\right\}_{i=1}^{n}$ and $\left\{F_{i}\right\}_{i=1}^{n}$ be the graph-directed sets of $G^{*}$ and $H^{*}$ respectively. Let $T_{e}$ and $S_{e}$ be the similitudes for $G^{*}$ and $H^{*}$ respectively. Suppose that

i) The base graphs coincide, i.e. $G=H$ and

ii) For each edge e of $G$, the similitudes $S_{e}$ and $T_{e}$ have the same ratio $\rho_{e}$;

iii) $\left\{E_{i}\right\}_{i=1}^{n}$ and $\left\{F_{i}\right\}_{i=1}^{n}$ are dust-like.

Then $E_{i}$ is bilipschitz equivalent to $F_{i}$ for all $1 \leq i \leq N$.

\section{Proof:}

[2] To begin, recall that $\operatorname{diam} E=\sup \{\mid x-y \| x, y \in E\}$. Also, set the distance between two sets, $A, B$ to be $\operatorname{dist}(A, B)=\inf \{\mid x-y \| x \in A, y \in B\}$ and let $T_{e_{1} \ldots e_{k}}=T_{e_{1}} \circ \ldots \circ T_{e_{k}}$.

Now, we are given that $\left\{E_{i}\right\}_{i=1}^{N}$ are dust-like, so for all $x \in E_{i}$ we can find a unique infinite path $e_{1} e_{2} e_{3} \ldots$ so that

$$
\{x\}=\bigcap_{k=1}^{\infty} T_{e_{1} e_{2} \ldots e_{k}}\left(E_{i_{k}}\right) .
$$

Note that $i_{k}$ is the vertex at the end of $e_{k}$. Now, $e_{1} e_{2} e_{3} \ldots$ is called a coding of $x$. Now look 
at $f: E_{i} \rightarrow F_{i}$ given by

$$
\{f(x)\}=\bigcap_{k=1}^{\infty} S_{e_{1} e_{2} \ldots e_{k}}\left(F_{i_{k}}\right) .
$$

It is clear that $f$ is one-to-one as well as onto. We just need to show that $f$ satisfies the bilipschitz condition.

Let $x, x^{\prime} \in E_{i}$. Let $e_{1} e_{2} e_{3} \ldots$ and $e_{1}^{\prime} e_{2}^{\prime} e_{3}^{\prime} \ldots$ be the codings for $x$ and $x^{\prime}$ respectively. Let $m \in \mathbb{Z}$ be the largest value with $e_{1} e_{2} e_{3} \ldots e_{m}=e_{1}^{\prime} e_{2}^{\prime} e_{3}^{\prime} \ldots e_{m}^{\prime}$. Now $x, x^{\prime} \in E_{i}$ so we have that

$$
\left|x-x^{\prime}\right| \leq \operatorname{diam} T_{e_{1} e_{2} \ldots e_{m}}\left(E_{i_{m}}\right) \leq \prod_{i=1}^{m} \rho_{e_{i}} \operatorname{diam}\left(E_{i_{m}}\right)
$$

Now, since $m$ is the largest value with $e_{1} e_{2} e_{3} \ldots e_{m}=e_{1}^{\prime} e_{2}^{\prime} e_{3}^{\prime} \ldots e_{m}^{\prime}$, we know that:

$$
\left|x-x^{\prime}\right| \geq d\left(T_{e_{1} e_{2} \ldots e_{m} e_{m+1}}\left(E_{i_{m+1}}\right), T_{e_{1} e_{2} \ldots e_{m} e_{m+1}^{\prime}}\left(E_{i_{m+1}^{\prime}}\right) \geq \prod_{i=1}^{m} \rho_{e_{i}} \min _{\left(e, e^{\prime}\right)} d\left(T_{e}\left(E_{j}\right), T_{e^{\prime}}\left(E_{j^{\prime}}\right)\right)\right.
$$

The minimum is taken over all pairs of edges, $\left(e, e^{\prime}\right)$, beginning at a common vertex, call it $i$. For $\left(e, e^{\prime}\right)$ the ending vertices are denoted by $j$ and $j^{\prime}$. Now, because $e$ and $e^{\prime}$ both begin at $i$ we know that $T_{e}\left(E_{j}\right) \cap T_{e}\left(E_{j}\right)=\emptyset$ as well as the fact that both sets are closed. This gives us that the minimum is larger than zero.

Thus, we know that there is a constant $c_{1}>0$ depending only on $G^{*}$ with

$$
c_{1}^{-1} \prod_{i=1}^{m} \rho_{e_{i}} \leq\left|x-x^{\prime}\right| \leq c_{1} \prod_{i=1}^{m} \rho_{e_{i}} .
$$

Similarly we can find a $c_{2}>0$ such that

$$
c_{2}^{-1} \Pi_{i=1}^{m} \rho_{e_{i}} \leq\left|f(x)-f\left(x^{\prime}\right)\right| \leq c_{2} \Pi_{i=1}^{m} \rho_{e_{i}} .
$$


This gives us that

$$
c_{1}^{-1} c_{2}^{-1}\left|x-x^{\prime}\right| \leq\left|f(x)-f\left(x^{\prime}\right)\right| \leq c_{1} c_{2}\left|x-x^{\prime}\right|
$$

Hence we have a bilipschitz map from $E_{i}$ onto $F_{i}$ and the two spaces are bilipschitz equivalent.

Now we can prove the following theorem discussed above. We will accomplish this goal by finding graph directed sets $G^{*}$ and $H^{*}$ created from the same graph $G$ with invariant sets $M$ and $M^{\prime}$ and the same similitude ratios. Thus by the above theorem, $M$ and $M^{\prime}$ will be bilipschitz equivalent.

\section{Theorem 4 [2]}

If $M=\frac{M}{5} \cup\left(\frac{M}{5}+\frac{2}{5}\right) \cup\left(\frac{M}{5}+\frac{4}{5}\right)$ and $M^{\prime}=\frac{M^{\prime}}{5} \cup\left(\frac{M^{\prime}}{5}+\frac{3}{5}\right) \cup\left(\frac{M^{\prime}}{5}+\frac{4}{5}\right)$ then $M$ is bilipschitz equivalent to $M^{\prime}$.

\section{Proof:}

[2] By definition we have that $M$ and $M^{\prime}$ are both self-similar. We will find a common directed graph for $M$ and $M^{\prime}$ and apply the previous theorem.

Let $M_{1}=M, M_{2}=M \cup(M+2)$, and $M_{3}=M \cup(M+2) \cup(M+4)$. Then we have that

$$
M_{1}=\frac{M}{5} \cup\left(\frac{M}{5}+\frac{2}{5}\right) \cup\left(\frac{M}{5}+\frac{4}{5}\right)=\frac{M_{1}}{5} \cup\left(\frac{M_{2}}{5}+\frac{2}{5}\right)
$$




$$
M_{2}=\left(\frac{M_{1}}{5}+2\right) \cup\left(\frac{M_{3}}{5}\right) \cup\left(\frac{M_{2}}{5}+\frac{12}{5}\right)
$$

and

$$
M_{3}=\left(\frac{M_{1}}{5}+4\right) \cup\left(\frac{M_{3}}{5}\right) \cup\left(\frac{M_{3}}{5}+2\right) \cup\left(\frac{M_{2}}{5}+\frac{22}{5}\right) .
$$

Look at the graph directed set $G^{*}$ pictured below.

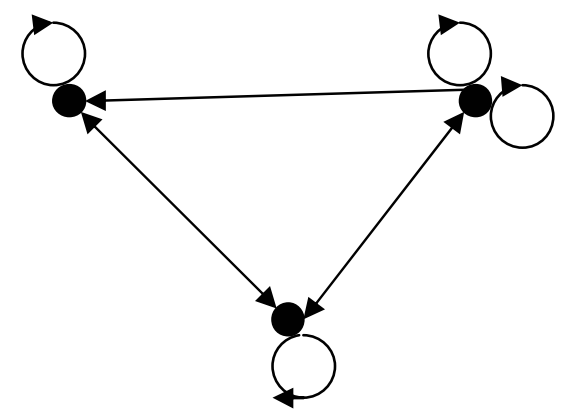

Figure 7.2: Directed Graph $G^{*}$

Label the vertices 1,2,3 counterclockwise, beginning at the top left. For vertex 1, the similitude paired with the loop is $\frac{1}{5} x$ and the edge connecting vertex 1 to vertex 2 is paired with the similitude $\frac{1}{5} x+\frac{2}{5}$. For vertex 2 , the loop is paired with the similitude $\frac{1}{5} x+\frac{12}{5}$, the edge connecting vertex 2 to vertex 3 is paired with $\frac{1}{5} x$ and the edge connecting vertex 2 to vertex 1 is paired with the similitude $\frac{1}{5} x+2$. For vertex 3 , one loop is paired with the similitude $\frac{1}{5} x$, one loop is paired with $\frac{1}{5} x+2$, the edge connecting vertex 3 with vertex 2 is paired with the similitude $\frac{1}{5} x+\frac{22}{5}$ and the edge connecting vertex 3 with vertex 1 is paired 
with $\frac{1}{5} x+4$.

Now,

$$
\begin{gathered}
M_{1}=\cup_{j=1}^{3} \cup_{T_{e} \in \Gamma_{1, j}} T_{e}\left(M_{j}\right)=\frac{M_{1}}{5} \cup\left(\frac{M_{2}}{5}+\frac{2}{5}\right) \\
M_{2}=\cup_{j=1}^{3} \cup_{T_{e} \in \Gamma_{2, j}} T_{e}\left(M_{j}\right)=\left(\frac{M_{1}}{5}+2\right) \cup\left(\frac{M_{3}}{5}\right) \cup\left(\frac{M_{2}}{5}+\frac{12}{5}\right)
\end{gathered}
$$

and

$$
M_{3}=\cup_{j=1}^{3} \cup_{T_{e} \in \Gamma_{3, j}} T_{e}\left(M_{j}\right)=\left(\frac{M_{1}}{5}+4\right) \cup\left(\frac{M_{3}}{5}\right) \cup\left(\frac{M_{3}}{5}+2\right) \cup\left(\frac{M_{2}}{5}+\frac{22}{5}\right) .
$$

Since the above unions are disjoint, we get that the invariant sets of $G^{*}$ are $\left\{M_{1}, M_{2}, M_{3}\right\}$, each of which are dust-like. Note that the similitude ratios are $\frac{1}{5}$ for each of the 9 similitudes.

Consider $M_{1}^{\prime}=M^{\prime}, M_{2}^{\prime}=M \cup\left(M^{\prime}+1\right)$, and $M_{3}^{\prime}=M^{\prime} \cup\left(M^{\prime}+1\right) \cup\left(M^{\prime}+2\right)$. Then we have that

$$
\begin{gathered}
M_{1}^{\prime}=\frac{M_{1}^{\prime}}{5} \cup\left(\frac{M_{2}^{\prime}}{5}+\frac{3}{5}\right) \\
M_{2}^{\prime}=\frac{M_{1}^{\prime}}{5} \cup\left(\frac{M_{3}^{\prime}}{5}+\frac{3}{5}\right) \cup\left(\frac{M_{2}^{\prime}}{5}+\frac{8}{5}\right)
\end{gathered}
$$

and 


$$
M_{3}^{\prime}=\frac{M_{1}^{\prime}}{5} \cup\left(\frac{M_{3}^{\prime}}{5}+\frac{3}{5}\right) \cup\left(\frac{M_{3}^{\prime}}{5}+\frac{8}{5}\right) \cup\left(\frac{M_{2}^{\prime}}{5}+\frac{13}{5}\right) .
$$

The similitudes in this case are as follows: $\frac{1}{5} x, \frac{1}{5} x+\frac{3}{5}, \frac{1}{5} x+\frac{3}{5}, \frac{1}{5} x+\frac{8}{5}, \frac{1}{5} x+\frac{3}{5}, \frac{1}{5} x+\frac{8}{5}$ and $\frac{1}{5} x+\frac{13}{5}$

Similarly, one can show $\left\{M_{1}^{\prime}, M_{2}^{\prime}, M_{3}^{\prime}\right\}$ are also invariant sets of $G^{*}$ and are also dust-like. As above, each similitude has similitude ratio $\frac{1}{5}$.

Thus, both $\left\{M_{1}, M_{2}, M_{3}\right\}$ and $\left\{M_{1}^{\prime}, M_{2}^{\prime}, M_{3}^{\prime}\right\}$ are dust-like and each similitude has ratio $\frac{1}{5}$. By the above theorem we have that $M_{1}$ is equivalent to $M_{1}^{\prime}$ and hence $M$ is equivalent to $M^{\prime}$ as desired.

What we have done in this proof is come up with two directed graphs that share the same base graph. $M$ as an invariant set of one directed graph and the other has $M^{\prime}$ as an invariant set. We also have all of the hypothesis of theorem 3 satisfied we achieved our desired result.

Now we give another example.

Theorem 5 Define subsets of $\mathbb{R}$ to be $M=\frac{M}{4} \cup\left(\frac{M}{4}+\frac{1}{2}\right)$ and $M^{\prime}=\frac{M^{\prime}}{4} \cup\left(\frac{M^{\prime}}{4}+\frac{3}{4}\right)$ then $M$ is bilipschitz equivalent to $M^{\prime}$.

\section{Proof:}

By definition we have that $M$ and $M^{\prime}$ are both self-similar. We will find directed graphs for both $M$ and $M^{\prime}$ and apply the previous theorem.

Let $M_{1}=M$ and $M_{2}=M \cup(M+2)$. Then we have that 


$$
M_{1}=\frac{M_{1}}{4} \cup \frac{M_{2}}{4}
$$

and

$$
M_{2}=\frac{M_{2}}{4}
$$

Look at the graph directed set $H^{*}$ pictured below.

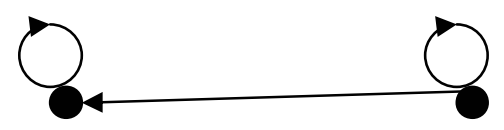

Figure 7.3: Directed Graph $H^{*}$

As before,our invariant sets of $H^{*}$ are $\left\{M_{1}, M_{2}\right\}$.

Consider $M_{1}^{\prime}=M^{\prime}$ and $M_{2}^{\prime}=M \cup\left(M^{\prime}+3\right)$. Then we have that

$$
M_{1}^{\prime}=\frac{M_{1}^{\prime}}{4} \cup \frac{M_{2}^{\prime}}{4}
$$

and

$$
M_{2}^{\prime}=\frac{M_{2}^{\prime}}{4}
$$

As before, $\left\{M_{1}^{\prime}, M_{2}^{\prime}\right\}$ are also invariant sets of $H^{*}$. Again, both $\left\{M_{1}, M_{2}\right\}$ and $\left\{M_{1}^{\prime}, M_{2}^{\prime}\right\}$ 
are dust-like and each similitude has ratio $\frac{1}{4}$. By the above theorem we have that $M_{1}$ is equivalent to $M_{1}^{\prime}$ and hence $M$ is equivalent to $M^{\prime}$ as desired. $\square$ 


\section{Chapter 8}

\section{Bilipschitz Equivalence of Cantor Sets}

\section{Continued}

In the last chapter we learned a way to prove bilipschitz equivalence of sets. The main theorem of this chapter provides properties invariant under bilipschitz equivalence and so provides a way to prove that sets are not bilipschitz equivalent. Restricted to Cantor sets, as in definition 11, these invariants give if and only if conditions for bilipschitz equivalence. To do this we will need to define several new terms, as in Falconer and Marsh's On the Lipschitz Equivalence of Cantor Sets [3] and follow the techniques given in that paper.

To begin this discussion we will introduce some new terms and notations. First let $V \subseteq \mathbb{R}^{d}$ where $d \in \mathbb{N}$. Let $m \in \mathbb{N}$ be larger than 2 and $\phi_{i}: \bar{V} \rightarrow \bar{V}$ be bijective maps which are also contractions, i.e, $d(f(x), f(y)) \leq r d(x, y)$ for $r<1$. We showed in chapter 5 that there is a 
unique, compact, invariant set $E \subseteq \bar{V}$ that is invariant. Recall the following definitions from chapter 5 .

Recall that, for $\phi_{i}$ as above we define a set $E \subseteq \bar{V}$ is called invariant if

$$
E=\bigcup_{i=1}^{m} \phi_{i}(E) .
$$

If all $\phi_{i}$ are similitudes then $E$ is called self-similar. If this is a disjoint union then $E$ is said to be dust-like [3].

Note that dust-like sets are totally disconnected.

During the study of invariant sets, one usually looks at how different functions, and compositions of those functions, affect invariant sets. Recall the following

A set of contractions $\left\{\phi_{i}\right\}_{i=1}^{m}$ is called an iterated function scheme [3].

Now, let $E$ be an invariant set with $E=\bigcup_{i=1}^{m} \phi_{i}(E)$. Then for $k \in \mathbb{Z}$ with $k \geq 0$ and let $\mathcal{M}_{k}=\left\{\left(i_{1}, i_{2}, \ldots, i_{k}\right) \mid 1 \leq i_{r} \leq m\right\}$. Let $\mathcal{M}_{\infty}=\left\{\left(i_{1}, i_{2}, \ldots\right) \mid 1 \leq i_{r} \leq m\right\}$ and let $\mathcal{M}_{0}=\emptyset$ Denote $\mathbf{i}=\left(i_{1}, i_{2}, \ldots, i_{k}\right)$ and $|\mathbf{i}|=k$ the length of the sequence. [3]

Definition 14 [3] $A$ cut set is a subset $\mathcal{J}$ of $\mathcal{M}=\cup_{k} \mathcal{M}_{k}$ so that for every $\left(i_{1}, i_{2}, \ldots\right) \in$ $\mathcal{M}_{\infty}$ there is a unique $k \in \mathbb{Z}$ with $\left(i_{1}, i_{2}, \ldots, i_{k}\right) \in \mathcal{J}$.

We will also write $\phi_{\mathbf{i}}=\phi_{i_{1}} \circ \phi_{i_{2}} \circ \ldots \circ \phi_{i_{k}}$ for $\mathbf{i}=\left(i_{1}, i_{2}, \ldots, i_{k}\right) \in \mathcal{M}_{k}$. Also, let $E_{\mathbf{i}}=E_{i_{1} i_{2} \ldots i_{k}}=$ $\phi_{\mathbf{i}}(E) .[3]$

Definition 15 [3] The basic sets of $E$ are $\left\{E_{\mathbf{i}}\right\}$ for $\mathbf{i} \in \mathcal{M}_{k}$. 
Lastly, we sometimes denote the single point $\bigcap_{k=1}^{\infty} E_{i_{1}, i_{2}, \ldots i_{k}}$ by $E_{\mathbf{i}}$ where $\mathbf{i} \in \mathcal{M}_{\infty}$. Note that the map from $\mathbf{i} \rightarrow E_{\mathbf{i}}$ is bijective since $E$ is dust-like. [3]

Now, assume that $E$ and $F$ are both dust-like and self-similar with the same Hausdorff dimension, say $s$. Now for the set $E$, let $\phi_{1}, \ldots, \phi_{m}$ be the similarity transformations with ratios $\alpha_{1}, \ldots, \alpha_{m}$ where $\Sigma_{1}^{m} \alpha_{i}=1$ and $0<\alpha_{i}<1$. As before let $\mathcal{M}_{k}=\left\{\left(i_{1}, \ldots 1_{k}\right) \mid 1 \leq i_{r} \leq\right.$ $m\}$ and $\mathcal{M}_{\infty}=\left\{\left(i_{1}, i_{2}, i_{3}, \ldots\right\}\right.$. Denote the basic sets of $E$ by $E_{\mathbf{i}}=\phi_{\mathbf{i}}(E)$ where $\mathbf{i} \in \mathcal{M}$. For the set $F$ let $\psi_{1}, \ldots, \psi_{n}$ be the similarity transformations with ratios $\beta_{1}, \ldots, \beta_{n}$ where $\sum_{1}^{n} \beta_{j}^{s}=1$ and $0<\beta_{j}<1$. Also, let $\mathcal{N}_{k}=\left\{\left(i_{1}, \ldots 1_{k}\right) \mid 1 \leq i_{r} \leq n\right\}$ and $\mathcal{N}_{\infty}=\cup \mathcal{N}_{k}$. Set the basic sets of $F$ to be $F_{\mathbf{j}}=\psi_{\mathbf{j}}(F)$ with $\mathbf{j} \in \mathcal{N}$. [3]

Before we state the next theorem we will need some new notation. Set $\mathbb{Q}\left(\alpha_{1}, \ldots, \alpha_{m}\right)$ to be the subfield of $(\mathbb{R},+, \times)$ generated by rational combinations of $\left(\alpha_{1}, \ldots, \alpha_{m}\right)$. Set $\operatorname{sgp}\left(\alpha_{1}, \ldots, \alpha_{m}\right)$ to be the sub-semigroup of $(\mathbb{R}, \times)$ generated by $\left(\alpha_{1}, \ldots, \alpha_{m}\right) .[3]$

Theorem 6 [3]Let $E$ and $F$ be bilipschitz equivalent dust-like self-similar sets of Hausdorff dimension s. Then we have

(i) $\mathbb{Q}\left(\alpha_{1}^{s}, \ldots, \alpha_{m}^{s}\right)=\mathbb{Q}\left(\beta_{1}^{s}, \ldots, \beta_{n}^{s}\right)$;

(ii) there exist $p, q \in \mathbb{N}$ with $\operatorname{sgp}\left(\alpha_{1}^{p}, \ldots, \alpha_{m}^{p}\right) \subseteq \operatorname{sgp}\left(\beta_{1}, \ldots, \beta_{n}\right)$ and $\operatorname{sgp}\left(\beta_{1}^{q}, \ldots, \beta_{n}^{q}\right) \subseteq$ $\operatorname{sgp}\left(\alpha_{1}, \ldots, \alpha_{m}\right)$.

\section{Proof:}

See Falconer and Marsh's On the Lipschitz Equivalence of Cantor Sets. 
Proposition 9 [3] The middle-third Cantor set, denoted by E, is bilipschitz equivalent to the self similar set $F$ described in $(i)$ if and only if condition (ii) holds:

(i) F is the set created by repeatedly replacing each interval by disjoint subintervals of positive lengths with ratios $\beta_{i}$ for $1 \leq i \leq n$ and

(ii) for $s=\frac{\ln (2)}{\ln (3)}$ as well as for each $i, \beta_{i}^{s}=2^{-k}$ for some $k=1,2,3, \ldots$ and $\Sigma_{1}^{n} \beta_{i}^{s}=1$.

\section{Proof:}

[3] Using the notation giving above we let $\alpha_{1}=\alpha_{2}=\frac{1}{3}$ and $s=\frac{\ln (2)}{\ln (3)}$. Let $E$ be bilipschitz equivalent to $F$. Then by the first part of the above theorem we have $\beta_{i}^{s} \in \mathbb{Q}\left(\frac{1}{2}, \frac{1}{2}\right)=\mathbb{Q}$. By the second part of the theorem we have that there exists $p \in \mathbb{N}$ so that $\beta_{1}^{s p} \in \operatorname{sgp}\left(\alpha_{1}^{s}, \alpha_{2}^{s}\right)=$ $\operatorname{sgp}\left(\frac{1}{2}\right)$ since $\frac{1}{3}^{s}=\frac{1}{3}^{\frac{\ln (2)}{\ln (3)}}=\frac{1}{2}$ by properties of the natural logarithm. So $\beta_{1}^{s p} \in \operatorname{sgp}\left(\frac{1}{2}\right)=$ $\left\{2^{-k} \mid k=0,1,2, \ldots\right\}$. This gives us that $2^{\frac{-k}{p}}=\beta_{1}^{s} \in \mathbb{Q}$. Thus we have $\frac{-k}{p} \in \mathbb{Z}$ and hence $\beta_{1}^{s}=2^{-l}$ where $l=0,1,2, \ldots$. Similarly, we can show $\beta_{2}^{s}, \ldots, \beta_{n}^{s}$ are all negative powers of 2. Since the Hausdorff dimension of $F$ is $s$ we get $\Sigma_{1}^{n} \beta_{i}^{s}=1$.

Assume that (i) and (ii) hold. Choose a cut set $\left\{\mathbf{i}_{\mathbf{1}}, \mathbf{i}_{\mathbf{2}}, \ldots \mathbf{i}_{\mathbf{n}}\right\} \in \mathcal{M}$ so that $\alpha_{\mathbf{i}_{j}}=\beta_{j}$ for $j=1,2, \ldots, n$. Then the map sending $E_{\mathbf{i}_{j(1)}, \mathbf{i}_{j(2)}, \ldots} \longleftrightarrow F_{j(1), j(2), \ldots}$ is a bilipschitz equivalence of $E$ and $F$. 


\section{Chapter 9}

\section{A Look Ahead}

There are many more directions to go in the study of the geometry of sets that are selfsimilar or "nearly' self-similar. We can study what occurs when we replace scaled isometry by scaled bilipschitz equivalence. We can also look at what happens when we replace the relationship between any two balls by a relationship between substantial subsets of any two balls. The latter is like the passage from "everywhere" to " almost everywhere" encountered in the passage from elementary real analysis to measure theory.

We can also look at different equivalence relations that we can place upon such sets. One possible equivalence relation is called BPI equivalence. BPI equivalence draws significance from being better adapted to sets that are "not quite" self-similar.

Before we learn what $B P I$ equivalence means we need to define a $C$-conformally bilipschitz map as well as Ahlfors dimension and a $B P I$ space. First, let us define a $C$-conformally 
bilipschitz map.

Definition 16 [1] Given two metric spaces $(M, d)$ and $(N, \rho)$ and a mapping $f: M \rightarrow N$ where $C^{-1} \lambda d(x, y) \leq \rho(f(x), f(y)) \leq C \lambda d(x, y)$ for some $\lambda>0$ and all $x, y \in M$ then we say that $M$ is $\mathbf{C - c o n f o r m a l l y ~ b i l i p s c h i t z ~ t o ~} N$ with scale factor $\lambda$.

Definition 17 A metric space $(M, d(x, y))$ is said to be (Ahlfors) regular of dimension $\mathbf{d}$ (or simply regular) if it is complete, has positive diameter, and if there is a constant $C>0$ such that

$$
C^{-1} r^{d} \leq H^{d}(B(x, r)) \leq C r^{d}
$$

for all $x \in M$ and $0<r \leq \operatorname{diam} M$. Here $H^{d}$ denotes the d-dimensional Hausdorff measure on $M$.

Now, the definition of a BPI space.

Definition $18[1]$ A metric space $(M, D)$ is called BPI space, or big pieces of itself if it is Ahlfors regular of dimension d, for any $d$, and if there exists constants $C, \theta$ so that for each $B(x, r)$ and $B(y, t)$ in our space $M$ where $0<r, t \leq \operatorname{diam} M$, there is a closed set $A$ such that $H^{d}(A) \geq \theta r^{d}$ and a map $h: A \rightarrow B(y, t)$ which is $C$-conformally bilipschitz with $\frac{t}{r}$ as the scale factor. 
Example 10 Let $F$ be a set with $k$ elements. Let $\lambda$ be a probability measure on $F$. Let $D$ be a metric on $F^{\infty}$ such that $D(x, y)=\Pi_{1}^{L(x, y)} \lambda\left(x_{i}\right)$, with $L(x, y)$ as before. $\left(F^{\infty}, D\right)$ is a BPI space.

This is shown in [1].

BPI spaces need not be completely self-similar. However, we can still define a relation that turns out to be an equivalence relation.

Definition 19 [1] Two BPI spaces $(M, d)$ and $(N, \rho)$ of the same dimension, say d, are said to be BPI equivalent if there exists $K, \alpha>0$ constants so that for all $x \in M$ and $0<r \leq \operatorname{diam} M$, as well as $u \in N$ and $0<t \leq \operatorname{diam} N$ there exists $A \subseteq B_{M}(x, r)$ closed where $H^{d}(A) \geq \alpha r^{d}$ and $\phi: A \rightarrow B_{N}(u, t)$ a K-conformally bilipschitz map with scale factor $\frac{t}{r}$.

BPI equivalence is actually an equivalence relation. To show this we need a few theorems from [1]:

Theorem 7 [1] If $(M, d)$ is a BPI metric space of dimension d, then any Ahlfors regular subset of dimension $d$ is also BPI of dimension $d$.

Theorem 8 [1] Let $(M, d)$ and $(N, \rho)$ are two BPI spaces with the same dimension. Then $M$ and $N$ are BPI equivalent if and only if there is $A \subseteq M$ with positive measure so that $A$ is bilipschitz equivalent to $B \subseteq N$. 
Theorem 9 [1] Let $M$ and $N$ be BPI spaces of the same dimension d.

a) If $M$ and $N$ are BPI equivalent, then there exists $\left\{A_{i}\right\}_{i=1}^{\infty}$, where each $A_{i}$ are closed in $M$, and bilipschitz maps $\phi_{i}: A_{i} \rightarrow N$ such that $N-\cup \phi_{i}\left(A_{i}\right)$ is of measure zero. The $\phi_{i}$ 's can be chosen such that they have uniformly bounded conformal bilipschitz constants. Also, each $A_{i}$ is a subset of a Borel subset of $M$ of positive measure.

b) If $M$ and $N$ are not BPI equivalent, and $A \subseteq M$ and $\phi: A \rightarrow N$ is bilipschitz then $H^{d}(A)=H^{d}(\phi(A))=0$.

Proposition 10 BPI equivalence is an equivalence relation.

\section{Proof:}

Let $A \equiv B$ denote $A$ BPI equivalent to $B$.

Reflexive:

This follows from the definition of BPI equivalence.

\section{Symmetric:}

Let $(M, d) \equiv(N, \rho)$, with both of dimension $d$. Then there exists $K, \alpha>0$ such that for all $x \in M, 0<r \leq \operatorname{diam} M, u \in N, 0<t \leq \operatorname{diam} N$ there exists a closed set $A \subseteq B_{M}(x, r)$ with $H^{d}(A) \geq \alpha r^{d}$ and a $K$-conformally bilipschitz mapping $\phi: A \rightarrow B_{N}(u, t)$ with scale factor $\frac{t}{r}$. 
Take $u \in N, x \in M, 0<t \leq \operatorname{diam} N$, and $0<r \leq \operatorname{diam} M$. Choose $K, \alpha, A \subseteq B_{M}(x, r)$ and $\phi$ as above.

Look at $\phi^{-1}: \phi(A) \rightarrow B_{M}(x, r)$. Let $\left\langle\phi\left(x_{n}\right)>\right.$ be a Cauchy sequence in $\phi(A)$. Then $<x_{n}>$ is a Cauchy sequence in $A$, since $A$ is closed as well as complete, $<x_{n}>\rightarrow x \in A$. So $<\phi\left(x_{n}\right)>\rightarrow \phi(x)$ and $\phi(A)$ is closed.

We know $K^{-1} \frac{t}{r} d(x, y) \leq \rho(\phi(x), \phi(y)) \leq K \frac{t}{r} d(x, y)$ for all $x, y \in A$. Hence we have that $K^{-1} d(x, y) \leq \frac{r}{t} \rho(\phi(x), \phi(y)) \leq K d(x, y)$.

Let $a, b \in \phi(A)$ with $\phi(x)=a, \phi(y)=b$. So

$$
K^{-1} d\left(\phi^{-1}(a), \phi^{-1}(b)\right) \leq \frac{r}{t} \rho(a, b) \leq K d\left(\phi^{-1}(a), \phi^{-1}(b)\right)
$$

and thus

$$
K^{-1} d\left(\phi^{-1}(a), \phi^{-1}(b)\right) \leq \frac{r}{t} \rho(a, b)
$$

and hence

$$
d\left(\phi^{-1}(a), \phi^{-1}(b)\right) \leq K \frac{r}{t} \rho(a, b)
$$

Also

$$
\frac{r}{t} \rho(a, b) \leq K d\left(\phi^{-1}(a), \phi^{-1}(b)\right)
$$

and thus we also know

$$
K^{-1} \frac{r}{t} \rho(a, b) \leq d\left(\phi^{-1}(a), \phi^{-1}(b)\right)
$$

Hence we have that

$$
K^{-1} \frac{r}{t} \rho(a, b) \leq d\left(\phi^{-1}(a), \phi^{-1}(b)\right) \leq K \frac{r}{t} \rho(a, b) .
$$


So $\phi^{-1}$ is a $K$-conformal bilipschitz map.

Now by Corollary 1 we have $H^{d}(\phi(A))>0$ since

$$
0<K^{-d} H^{d}(A) \leq H^{d}(\phi(A)) \leq K^{d} H^{d}(A) .
$$

Also by Corollary 1 and $H^{d}(A) \geq \alpha r^{d}$ we have that

$$
\begin{gathered}
H^{d}(\phi(A)) \geq K^{-d} \frac{t^{d}}{r} H^{d}(A) \\
\geq K^{-d} \alpha r^{d} \frac{t^{d}}{r} H^{d}(A) \\
\geq K^{-d} \alpha r^{d} \frac{t^{d}}{r} \\
=K^{-d} \alpha t^{d}
\end{gathered}
$$

Thus we have symmetry.

\section{Transitive:}

To show transitivity we must first prove the following lemma: 
Lemma 3 Let $(N, \rho)$ be a BPI space of dimension $d$. Let $B, B^{\prime} \subseteq N$ of positive measure. Then $B$ is BPI equivalent to $B^{\prime}$.

\section{Proof:}

Now $H^{d}(B)>0$ and $H^{d}\left(B^{\prime}\right)>0$ so both have dimension $d$, so it is possible for $B \equiv B^{\prime}$. By theorem $7, B$ and $B^{\prime}$ are BPI. Using identity mappings it is clear $B \equiv N$ and $B^{\prime} \equiv N$.

By theorem 9a, the exists $B_{i} \subseteq B$ that are closed, $\phi_{i}: B_{i} \rightarrow N$ with $H^{d}\left(N-\cup_{i} \phi_{i}\left(B_{i}\right)\right)=0$ and $\phi_{i}$ bilipschitz with uniform bounded constants. Since $H^{d}\left(N-\cup_{i} \phi_{i}\left(B_{i}\right)\right)=0$ we get that $H^{d}\left(B^{\prime}-\cup_{i} \phi_{i}\left(B_{i}\right)\right)=0$. So $H^{d}\left(B^{\prime} \backslash \cap_{i} \phi_{i}\left(B_{i}\right)\right)=H^{d}\left(B^{\prime}\right)>0$. Thus there exists $i$ such that $\left|\phi_{i}\left(B_{i}\right) \cap B^{\prime}\right|>0$, where $|A|$ denotes the measure of $A$. Set $\phi=\left.\phi_{i}\right|_{\phi_{i}^{-1}\left(B_{i}\right)}$ for our chosen value of $i$.

So $\phi: \phi_{i}^{-1}\left(B^{\prime}\right) \rightarrow \phi\left(B_{i}\right) \cap B^{\prime}$ and $\phi: \phi_{i}^{-1}\left(B^{\prime}\right) \rightarrow B^{\prime}$, is bilipschitz.

Suppose $B \not \equiv B^{\prime}$. Then by theorem $9 \mathrm{~b}$ we get $\phi^{-1}\left(B^{\prime}\right) \subseteq B$ and $\phi: \phi^{-1}\left(B^{\prime}\right) \rightarrow B^{\prime}$ is bilipschitz, hence $H^{d}\left(\phi^{-1}\left(B^{\prime}\right)\right)=H^{d}\left(\phi\left(B_{i}\right) \cap B^{\prime}\right)=0$. However, by choice of $i, H^{d}\left(\phi\left(B_{i}\right) \cap\right.$ $\left.B^{\prime}\right)>0$, a contradiction.

Hence $B \equiv B^{\prime}$.

Now to show transitivity:

Let $M \equiv N \equiv P$. Then by theorem $8, \exists A \subseteq M, B \subseteq N$ such that $H^{d}(A)>0$ and $A$ is bilipschitz equivalent to $B$ with mapping $\phi$. (This gives that $H^{d}(B)>0$ by Corollary 1. ) Also by theorem 8 we get that there exits $B^{\prime} \subseteq N, C \subseteq N$ such that $H^{D}\left(B^{\prime}\right)>0$ and $B^{\prime}$ is 
bilipschitz equivalent to $C$ with mapping $\psi$. (This gives $H^{d}(C)>0$.)

Now by lemma 3 we have that $B \equiv B^{\prime}$. So there is a subset $D \subset B$ with $H^{d}(D)>0$ bilipschitz equivalent to $D, \subseteq B^{\prime}$, by mapping $f$.

Thus we have that

$A \longleftrightarrow B \supseteq D \longleftrightarrow D^{\prime} \subseteq B^{\prime} \longleftrightarrow C$ with

$\phi: A \rightarrow B, f: D \rightarrow D^{\prime}$ and $\psi: B^{\prime} \rightarrow C$.

Let $A^{\prime}=\phi^{-1}(D)$ which has $H^{d}\left(A^{\prime}\right)>0$. Let $C^{\prime}=\psi\left(D^{\prime}\right)$, again which has positive measure. Thus $\phi: A^{\prime} \rightarrow D, F: d \rightarrow D^{\prime}$ and $\psi: D^{\prime} \rightarrow C^{\prime}$ are all onto bilipschitz mappings. Thus $\phi \circ f \circ \psi$ is an onto bilipschitz mapping of $A^{\prime}$ onto $C^{\prime}$. Thus we know that $A^{\prime}$ is bilipschitz equivalent to $C^{\prime}$ and $H^{d}\left(A^{\prime}\right)>0$. Thus $M \equiv P$ by theorem 8 .

Notice that showing that BPI equivalence is an equivalence relation is much harder than showing that bilipschitz equivalence is an equivalence relation. BPI equivalence is far more complicated than bilipschitz equivalence. If follow the construction of example 10 but vary the probability measure of $\mathrm{F}$, we define a $(k-1)$-dimensional family of BPI spaces. Although the sets constructed in this way are actually "completely" self-similar sets, which of them are BPI equivalent is an open question [1].

We could also generalize example 10 by placing a sequence $\left(\lambda_{i}\right)$ of probability measures on $F^{\infty}$. The new metric on $F^{\infty}$ is defined by $d(x, y)=\Pi_{1}^{L(x, y)} \lambda_{i}\left(x_{i}\right)$.

Let $\left(F^{\infty}, D\right)$ be as defined above. Let $\left\{\lambda_{i}\right\}$ be periodic with period $p$. Let us show that any 
two balls have sub-balls which are related by a scaled isometry.

Take $B\left(x, \lambda_{1}\left(x_{1}\right) \lambda_{2}\left(x_{2}\right) \ldots \lambda_{k}\left(x_{k}\right)\right)$ and $B\left(y, \lambda_{1}\left(y_{1}\right) \lambda_{2}\left(y_{2}\right) \ldots \lambda_{n}\left(y_{n}\right)\right)$ be open balls in $F$. Set $r=\lambda_{1}\left(x_{1}\right) \lambda_{2}\left(x_{2}\right) \ldots \lambda_{k}\left(x_{k}\right)$ and $t=\lambda_{1}\left(y_{1}\right) \lambda_{2}\left(y_{2}\right) \ldots \lambda_{n}\left(y_{n}\right)$

Let $A=\bar{B}\left(\left(x, \lambda_{1}\left(x_{1}\right) \lambda_{2}\left(x_{2}\right) \ldots \lambda_{k}\left(x_{k}\right) \ldots \lambda_{k+l}\left(x_{k+l}\right)\right)\right.$, where $k+l$ is equivalent to $n \bmod -$ ulo $p$. Note that $A$ is a closed set. Let $a, b \in A$. Let $f: A \rightarrow B(y, t)$ with $f(a)=$ $y_{1}, y_{2}, \ldots, y_{n} a_{k+l+1}, a_{k+l+2} \ldots$

We have that

$$
D(a, b)=\Pi_{1}^{L(a, b)} \lambda_{i}\left(a_{i}\right)=\Pi_{1}^{k+l+1} \lambda_{i}\left(x_{i}\right) \Pi_{k+l+1}^{L(a, b)} \lambda_{i}\left(a_{i}\right)
$$

Also, we have that

$$
\Pi_{1}^{n} \lambda_{i}\left(y_{i}\right) \Pi_{n+1}^{L(f(a), f(b))} \lambda_{i}\left(f\left(a_{i}\right)\right)=\Pi_{1}^{n} \lambda_{i}\left(y_{i}\right) \Pi_{n+1}^{L(a, b)} \lambda_{i}\left(f(a)_{i}\right)
$$

Since $n$ is equivalent to $k+l$ modulo $p$ we have that

$$
\Pi_{n+1}^{L(f(a), f(b))} \lambda_{i}\left(f(a)_{i}\right)=\Pi_{k+l+1}^{L(a, b)} \lambda_{i}\left(a_{i}\right)
$$

Thus, we have that

$$
\frac{t}{r} D(a, b)=\Pi_{1}^{k+l+1} \lambda_{i}\left(x_{i}\right) \Pi_{k+l+1}^{L(a, b)} \lambda_{i}\left(a_{i}\right)=t \Pi_{k+l+1}^{L(a, b)} \lambda_{i}\left(a_{i}\right)=\Pi_{1}^{n} \lambda_{i}\left(y_{i}\right) \Pi_{k+l+1}^{L(a, b)} \lambda_{i}\left(a_{i}\right)
$$

Thus,

$$
\frac{t}{r} D(a, b)=\Pi_{1}^{n} \lambda_{i}\left(y_{i}\right) \Pi_{k+l+1}^{L(a, b)} \lambda_{i}\left(a_{i}\right)=\Pi_{n+1}^{L(f(a), f(b))} \lambda_{i}\left(f(a)_{i}\right)=D(f(a), f(b)) .
$$


Therefore,

$$
\frac{t}{r} D(a, b) \leq D(f(a), f(b)) \leq \frac{t}{r} D(a, b)
$$

In this proof we have shown that, for any two balls, there are sub-balls which are related by a scaled isometry. This is because the interior of our closed set $A$ is an open sub-ball of $B(x, r)$ which is related to $B(y, t)$ by a scaled isometry. This is due to the periodicity of the probability measures. Without the periodicity we could not define our function $f$ so that $\Pi_{1}^{n} \lambda_{i}\left(y_{i}\right) \Pi_{k+l+1}^{L(a, b)} \lambda_{i}\left(a_{i}\right)=\Pi_{n+1}^{L(f(a), f(b))} \lambda_{i}\left(f(a)_{i}.\right)$

Also note, since the probability measures are periodic, $\left(F^{\infty}, D\right)$ is self-similar. Let $B=$ $\left\{\left(a_{1}, a_{2}, \ldots, a_{p}\right) \mid 1 \leq a_{l} \leq k\right\}$. Set the cardinality of $B$ to be $r$. Let $f: B \rightarrow B$ be a bijection. Then $F=\cup_{1}^{r} \phi_{j}(F)$ with $\phi_{j}\left(a_{1}, a_{2}, \ldots\right)=\left(f(j), a_{1}, a_{2}, \ldots\right) . \phi_{j}$ is a similitude with similitude ratio $\Pi_{m=1}^{p} \lambda_{m}\left(f(j)_{m}\right)$. Note, for $\phi_{j}, D\left(\phi_{j}(a), \phi_{j}(b)\right)=\Pi_{m=1}^{p} \lambda_{m}\left(f(j)_{m}\right) D(a, b)$.

It turns out that if the probability measures are periodic then $\left(F^{\infty}, D\right)$ is a BPI space. However, we do not good general sufficient conditions to show $\left(F^{\infty}, D\right)$ is a BPI space or conditions that determine when two such spaces are BPI equivalent.

We hope try to extend results about bilipschitz equivalence discussed in this paper to the setting of BPI equivalence. For example, we could try proving sets are BPI equivalent by using graph-theoretic techniques. We could also attempt to find invariants under BPI equivalence. This could lead to showing certain sets are not BPI equivalent. The construction of example 10, applied with different probability measures is a source of examples against 
which to test these ideas. The same is true of the generalized construction base on sequences of probability measure. 


\section{Bibliography}

[1] G. David and S. Semmes, Fractured Fractals and Broken Dreams, Oxford University Press, New York, 1997.

[2] H. Rao, H. J. Ruan, L. F. Xi, Lipschitz equivalence of self-similar sets C.R. Acad. Sci. Paris, Ser. I 342 (2006), 191-196.

[3] K. Falconer, D. Marsh, On the Lipschitz Equivalence of Cantor Sets, Mathematika, 39 (1992), 223-233.

[4] K. Falconer, Fractal Geometry, Mathematical Foundations and Applications, John Wiley \& Sons, Chichester, 1990. 